\title{
Forged in FIRE: cusps, cores and baryons in low-mass dwarf galaxies
}

\author{
Jose Oñorbe, ${ }^{1,2 \star}$ Michael Boylan-Kolchin, ${ }^{3,4}$ James S. Bullock, ${ }^{1}$ Philip F. Hopkins, ${ }^{5}$ \\ Dušan Kereš, ${ }^{6}$ Claude-André Faucher-Giguère, ${ }^{7}$ Eliot Quataert ${ }^{8}$ and Norman Murray ${ }^{9}$ \\ ${ }^{1}$ Department of Physics and Astronomy, University of California at Irvine, Irvine, CA 92697, USA \\ ${ }^{2}$ Max-Planck-Institut für Astronomie, Koenigstuhl 17, D-69117 Heidelberg, Germany \\ ${ }^{3}$ Department of Astronomy and Joint Space-Science Institute, University of Maryland, College Park, MD 20742-2421, USA \\ ${ }^{4}$ Department of Astronomy, The University of Texas at Austin, 2515 Speedway, Stop C1400, Austin, TX 78712, USA \\ ${ }^{5}$ TAPIR, Mailcode 350-17, California Institute of Technology, Pasadena, CA 91125, USA \\ ${ }^{6}$ Department of Physics, Center for Astrophysics and Space Science, University of California at San Diego, 9500 Gilman Drive, La Jolla, CA 92093, USA \\ ${ }^{7}$ Department of Physics and Astronomy and CIERA, Northwestern University, 2145 Sheridan Road, Evanston, IL 60208, USA \\ ${ }^{8}$ Department of Astronomy and Theoretical Astrophysics Center, University of California Berkeley, Berkeley, CA 94720, USA \\ ${ }^{9}$ Canadian Institute for Theoretical Astrophysics, 60 St. George Street, University of Toronto, ON M5S 3H8, Canada
}

Accepted 2015 September 4. Received 2015 August 27; in original form 2015 February 6

\begin{abstract}
We present multiple ultrahigh resolution cosmological hydrodynamic simulations of $M_{\star} \simeq$ $10^{4-6.3} \mathrm{M}_{\odot}$ dwarf galaxies that form within two $M_{\mathrm{vir}}=10^{9.5-10} \mathrm{M}_{\odot}$ dark matter halo initial conditions. Our simulations rely on the Feedback in Realistic Environments (FIRE) implementation of star formation feedback and were run with high enough force and mass resolution to directly resolve structure on the $\sim 200 \mathrm{pc}$ scales. The resultant galaxies sit on the $M_{\star}$ versus $M_{\text {vir }}$ relation required to match the Local Group stellar mass function via abundance matching. They have bursty star formation histories and also form with half-light radii and metallicities that broadly match those observed for local dwarfs at the same stellar mass. We demonstrate that it is possible to create a large $(\sim 1 \mathrm{kpc})$ constant-density dark matter core in a cosmological simulation of an $M_{\star} \simeq 10^{6.3} \mathrm{M}_{\odot}$ dwarf galaxy within a typical $M_{\mathrm{vir}}=10^{10} \mathrm{M}_{\odot}$ halo precisely the scale of interest for resolving the 'too big to fail' problem. However, these large cores are not ubiquitous and appear to correlate closely with the star formation histories of the dwarfs: dark matter cores are largest in systems that form their stars late $(z \lesssim 2)$, after the early epoch of cusp building mergers has ended. Our $M_{\star} \simeq 10^{4} \mathrm{M}_{\odot}$ dwarf retains a cuspy dark matter halo density profile that matches that of a dark-matter-only run of the same system. Though ancient, most of the stars in our ultrafaint form after reionization; the ultraviolet field acts mainly to suppress fresh gas accretion, not to boil away gas that is already present in the protodwarf.
\end{abstract}

Key words: methods: numerical-galaxies: dwarf-galaxies: evolution-galaxies: formation-cosmology: theory.

\section{INTRODUCTION}

Many of the most pressing problems associated with the standard $\Lambda$ cold dark matter $(\Lambda \mathrm{CDM})$ paradigm concern the faintest $M_{\star} \simeq 10^{6} \mathrm{M}_{\odot}$ dwarf galaxies and the dark matter haloes that have the right abundance to host them: $M_{\text {vir }} \simeq 10^{10} \mathrm{M}_{\odot}$ (Brook et al. 2014; Garrison-Kimmel et al. 2014a). If $\Lambda \mathrm{CDM}$ is correct, then the dark matter haloes hosting these dwarfs must be extremely inefficient at converting baryons into stars (Klypin et al. 1999) and they also must be significantly less dense in their centres than predicted in dissipationless $\Lambda \mathrm{CDM}$ simulations (Boylan-Kolchin, Bullock \&

^E-mail: onorbe@mpia.de
Kaplinghat 2011, 2012; Ferrero et al. 2012; Garrison-Kimmel et al. 2014b; Klypin et al. 2014; Tollerud, Boylan-Kolchin \& Bullock 2014; Papastergis et al. 2015). This latter issue (known as the 'too big to fail' problem) may be related to indications that dwarf galaxies reside within dark matter haloes that have cored density profiles rather than the cuspy Navarro-Frenk-White (NFW)-like profiles predicted in CDM simulations (Flores \& Primack 1994; de Blok et al. 2008; Kuzio de Naray, McGaugh \& de Blok 2008; Oh et al. 2008; Walker \& Peñarrubia 2011; Salucci et al. 2012; Amorisco, Zavala \& de Boer 2014; Ogiya \& Burkert 2015, but see Strigari, Frenk \& White 2014).

While some authors have taken these discrepancies as motivation to explore non-standard dark matter models (Macciò \& Fontanot 2010; Vogelsberger, Zavala \& Loeb 2012; Rocha et al. 2013; 
Horiuchi et al. 2014b; Governato et al. 2015), others have argued that it may be possible to naturally resolve them through a better understanding of star formation and feedback in low-mass galaxies. Specifically, the inefficiency of dwarf galaxy formation is believed to be driven by supernovae ( $\mathrm{SNe}$ ) feedback and the effects of an ionizing background (Dekel \& Silk 1986; Bullock, Kravtsov \& Weinberg 2000). Likewise, dark matter haloes may be transformed from cusps into cores if enough energy can be injected into the orbits of dark matter particles during rapid starburst events (Navarro, Eke \& Frenk 1996; Governato et al. 2010; Pontzen \& Governato 2012; Ogiya \& Mori 2014). As pointed out by Peñarrubia et al. (2012), these two requirements are at odds with each other: the need to lower the efficiency of star formation means that there will be less $\mathrm{SNe}$ energy available to create dark matter cores. Solving the two problems simultaneously therefore represents a significant theoretical challenge (Garrison-Kimmel et al. 2013).

Reproducing even the broad-brush properties of dwarfs in a cosmological framework, regardless of their internal structure, has been historically challenging. At these scales, the relationship between stellar mass and halo mass derived from local galaxy counts (Brook et al. 2014; Garrison-Kimmel et al. 2014b) implies a suppression of galaxy formation by a factor of about 1000 . While it is generally believed that stellar feedback is the main agent responsible for this suppression, actually getting a physically realistic model of the relevant processes to manifest these expectations has proven difficult.

The past several years have proven fruitful in this regard, with many published studies achieving substantial suppression in the conversion of baryons to stars on the scale of dwarf galaxy haloes (Governato et al. 2010, 2015; Sawala et al. 2011; Munshi et al. 2013; Simpson et al. 2013; Trujillo-Gomez et al. 2015). As we show below, however, many of these studies have not quite reached the level of suppression that seems to be required by local galaxy counts. Moreover, whether or not these feedback models also match the different observed scaling relations for these systems (Wolf et al. 2010; Kirby et al. 2013; Collins et al. 2014) is still not clear. Reproducing both the correct stellar mass and structural properties has proven to be an even more difficult challenge (Sales et al. 2010). The observed stellar metallicity-stellar mass tight correlation (Gallazzi et al. 2005; Kirby et al. 2013) can also put very important constraints on the feedback models and how these are implemented.

As for the question of feedback-driven core formation, much remains debated. Some of the most successful simulations at producing cores in dwarf galaxies have suggested a transition mass below $M_{\star} \sim 10^{7} \mathrm{M}_{\odot}$ where core formation becomes difficult (Governato et al. 2012). Using a slightly different set of simulations, Di Cintio et al. (2014) find similar results, and suggest that the cusp-core transition should be most effective when the ratio of stellar mass to dark matter halo mass relatively high, in massive dwarfs with $M_{\star} \sim 10^{8} \mathrm{M}_{\odot}$ and $M_{\text {vir }} \sim 3 \times 10^{10.5} \mathrm{M}_{\odot}$. Importantly, they also find that cuspy profiles are retained for the $M_{\star} \simeq 10^{6} \mathrm{M}_{\odot}$ dwarfs of concern (residing in $M_{\text {vir }}=10^{10} \mathrm{M}_{\odot}$ haloes) although resolution may have been an issue in these cases. At some mass scale, galaxy formation may become effectively stochastic (e.g. Boylan-Kolchin et al. 2011). Recent work by Sawala et al. (2014, 2015), however, suggests that the scale at which stochasticity becomes important is somewhat lower $\left(M_{\text {vir }} \sim 10^{9}-10^{9.5} \mathrm{M}_{\odot}\right)$.

Though the results of Di Cintio et al. (2014) and Governato et al. (2012) agree reasonably well, a different set of high-resolution simulations with a simpler implementation of stellar feedback have not produced cores in dwarf galaxy haloes at any mass (Vogelsberger et al. 2014), even though a number of other observables are well matched. The absence of cores produced by stellar feedback in these simulations could be due to the fact that their subgrid interstellar medium (ISM) and star formation model leads to star formation histories that are (likely) artificially smoothed in time, compared to the bursty star formation histories found in more explicit models (Hopkins et al. 2014a; Muratov et al. 2015). Conversely, TrujilloGomez et al. (2015) found that radiation pressure from massive stars was the most important source of core formation in their simulations, not thermal feedback from $\mathrm{SN}$, which has been the primary mode used by other groups that have produced cores. More generally, models for feedback that have been used up until now have been subgrid and necessitated ad hoc approximations, such as turning off cooling for material heated by SNe. As such it is not clear whether the feedback we actually expect from stellar evolution models is capable of producing large cores, or whether the mass limit for core formation is robust.

In this paper, we attempt to minimize the freedom of subgrid galaxy formation models and to incorporate as many important physical processes in a manner that is as realistic as possible at present in order to understand if and how star formation affects the gravitational potential wells of dwarf dark matter haloes. To these ends, we have conducted a series of high-resolution cosmological hydrodynamical simulations of two dwarf haloes using the code presented in Hopkins et al. (2014a). In this work, we showed that this implementation of stellar feedback successfully reproduces the observationally inferred relationship between the stellar mass-dark matter halo mass $\left(M_{\star}-M_{\text {halo }}\right)$ and star formation histories of galaxies at all redshifts where observational constraints are currently available. Faucher-Giguère et al. (2015) recently showed that it also replicates the neutral hydrogen content of high-redshift haloes.

To our knowledge, the set of simulation presented here include the current highest resolution simulation of this type with an explicit implementation of feedback yet achieved. This not only facilitates a more accurate treatment of astrophysical processes but is also crucial in the context of dwarfs as dark matter probes. The dwarfs of concern have half-light radii of $\sim 500 \mathrm{pc}$, and thus any dark matter core of relevance needs to be dynamically resolved at this scale. According to well-documented convergence test studies (Power et al. 2003), many previous simulations that have reported core formation on this scale were quite poorly resolved, some at only $\sim 2-3$ softening lengths. In what follows we make every effort to clarify our resolution limitations.

The paper is organized as follows. In Section 2, we describe the computational methods which we have used and our choice of initial conditions. We present the results of our simulations in Section 3. We pay closer attention to the matter content of our simulated dwarfs, and the possible formation of cores in Section 4. We conclude with a summary where we discuss the achievements and shortcomings of the simulations in Section 5.

\section{SIMULATIONS}

We have run a series of multimass cosmological hydrodynamical simulations (Porter 1985; Katz \& White 1993) following the formation and evolution of structure in the $\Lambda \mathrm{CDM}$ model of two dwarf galaxy haloes. Each simulation is a cosmological zoom-in that includes high-resolution gas and dark matter for the flow converging region that generates the main object. The rest of the simulation box is sampled by low-resolution dark matter particles that account for tidal forces. The cosmological model adopted throughout this paper is based on cosmic microwave background results 
(Komatsu et al. 2011): $\sigma_{8}=0.801, \Omega_{\Lambda}=0.734, \Omega_{\mathrm{m}}=0.266, \Omega_{\mathrm{b}}$ $=0.0449, n_{\mathrm{s}}=0.963$, and $h=0.71$.

To generate the cosmological initial conditions we made use of MUSIC, an OPENMP parallel algorithm to generate multiscale initial conditions with multiple levels of refinements for cosmological 'zoom' simulations (MUSIC; Hahn \& Abel 2011) and we followed the method outlined in Oñorbe et al. (2014). To select our dwarf candidates we first run a medium-resolution dark-matter-only cosmological simulation using GADGET-2 (Springel 2005) with a cubic volume of $7 \mathrm{Mpc}$ on a side with particle mass $m_{\mathrm{p}}=9.7 \times 10^{4} \mathrm{M}_{\odot}$ and Plummer equivalent force softening length of $176 \mathrm{pc}$. To be able to study the main statistical properties of dwarf galaxy haloes we also run a bigger dark-matter-only simulation of $35 \mathrm{Mpc}$ on a side with particle mass $m_{\mathrm{p}}=1.2 \times 10^{7} \mathrm{M}_{\odot}$ and Plummer equivalent force softening length of $563 \mathrm{pc}$. In this work we present simulations of two dwarf galaxy haloes, one with a virial mass of $M_{\text {vir }}=3.2 \times 10^{9} \mathrm{M}_{\odot}$ and the other with $M_{\text {vir }}=9.2 \times 10^{9} \mathrm{M}_{\odot} \cdot{ }^{1}$ Based on our analysis of the $35 \mathrm{Mpc}$ simulation, we have chosen our dwarf candidates to lie as close as possible to the mean values of spin, concentration, and halo formation time for its mass while still having a small Lagrangian volume (see Oñorbe et al. 2014). The specific values of these parameters for our two haloes can be found in Table 1. We point to Appendix A for a more detailed description of these parameters and how they compare with a sample of haloes in the same mass bin.

To check the convergence of our results we have run two resolution levels for our simulations: in our low-resolution hydrodynamical testing runs we use a dark matter particle mass of $1.01 \times$ $10^{4} \mathrm{M}_{\odot}$ and a particle gas mass of $2.04 \times 10^{3} \mathrm{M}_{\odot}$ (the mass resolution for the collisionless run is therefore $\left.1.22 \times 10^{4} \mathrm{M}_{\odot}\right)$. The high-resolution runs used a dark matter particle mass of $1.26 \times$ $10^{3} \mathrm{M}_{\odot}$ and a gas particle mass of $254 \mathrm{M}_{\odot}$ (the particle resolution for the collisionless run is therefore $1.5 \times 10^{3} \mathrm{M}_{\odot}$ ). None of the high-resolution regions of the simulations presented in this work is contaminated by low-resolution particles at any redshift within 1.6 virial radii.

The simulations presented in this paper use GIZMO $^{2}$ (Hopkins 2015), run in P-SPH mode which includes physical models for star formation and stellar feedback presented in Hopkins et al. (2014a). Two of the runs presented here ULTRAFAINT and DWARF_EARLY were also presented in Hopkins et al. (2014a) (m09 and m10, respectively). We summarize their properties below, but readers interested in further details (including resolution studies and a range of tests of the specific numerical methodology) should see Hopkins, Quataert \& Murray (2012) and Hopkins et al. (2013, 2014a).

For the halo identification in the simulation we have used the public code Amiga Halo Finder (AHF; Knollmann \& Knebe 2009), an MPI parallel code for finding gravitationally bound structures in simulations of cosmic structure. Results presented in this work use a highest density peak+sigma-clipping method to find the centre. We have also tested different centring algorithms to confirm that our results do not depend on which method was used. ${ }^{3}$

\footnotetext{
${ }^{1}$ Unless otherwise stated, in this paper we define the virial overdensity using the spherical top hat collapse approximation by Bryan \& Norman (1998).

${ }^{2}$ http://www.tapir.caltech.edu/phopkins/Site/GIZMO

${ }^{3}$ A simple centre-of-mass algorithm was the only method that we found not able to track the centre of our systems with the accuracy required for this work.
}

\subsection{Numerical methods}

The P-SPH method adopts the Lagrangian 'pressure-entropy' formulation of the smoothed particle hydrodynamics (SPH) equations developed in Hopkins (2013); this eliminates the major differences between SPH, moving mesh, and grid (adaptive mesh) codes, and resolves the well-known issues with fluid mixing instabilities in previously used forms of SPH (e.g. Agertz et al. 2007; Sijacki et al. 2012). P-SPH also manifestly conserves momentum, energy, angular momentum, and entropy. The gravity solver is a heavily modified version of the GADGET-3 (Springel 2005) hybrid tree-particle mesh (Tree-PM) method; but GIZMo also includes substantial improvements in the artificial viscosity, entropy diffusion, adaptive timestepping, smoothing kernel, and gravitational softening algorithm, as compared to the 'previous generation' of SPH codes. These are all described in detail in Hopkins et al. (2014a) and Hopkins (2015). In particular, in 'traditional' GADGET, softenings are not adaptive, and pairwise interactions are simply smoothed by the larger of the two particle softenings. We have also modified the softening kernel as described therein to represent the exact solution for the potential of the SPH smoothing kernel. Therefore our 'standard' simulations use adaptive gravitational softening lengths for gas which minimum is a factor of $\sim 10$ smaller than the fixed dark matter gravitational softening lengths. In order to test this approach we have also run the same initial conditions using identical softenings for both the baryonic and dark matter particles (close to the higher dark matter default value). We labelled these runs according to the late star formation history of the high-resolution runs (see Table 1 and the discussion below for more details).

In our simulations, gas follows an ionized+atomic + molecular cooling curve from 10 to $10^{10} \mathrm{~K}$, including metallicity-dependent fine structure and molecular cooling at low temperatures, and high-temperature $\left(\gtrsim 10^{4} \mathrm{~K}\right)$ metal line cooling followed speciesby-species for 11 separately tracked species. At all times, the appropriate ionization states and cooling rates are tabulated from a compilation of CLOUDY runs, including the effect of a uniform but redshift-dependent photoionizing background computed in Faucher-Giguère et al. (2009), ${ }^{4}$ together with local sources of photoionizing and photoelectric heating. Self-shielding is accounted for with a local Sobolev/Jeans length approximation (integrating the local density at a given particle out to a Jeans length to determine a surface density $\Sigma$, then attenuating the background seen at that point by $\left.\exp \left(\kappa_{v} \Sigma\right)\right)$.

Star formation is allowed only in dense, molecular, selfgravitating regions above $n>n_{\text {crit }}\left(n_{\text {crit }}=100 \mathrm{~cm}^{-3}\right.$ for our highresolution simulations). ${ }^{5}$ This threshold is much higher than that adopted in most 'zoom-in' simulations of galaxy formation (the high value allows us to capture highly clustered star formation). We follow Krumholz \& Gnedin (2011) to calculate the molecular fraction $f_{\mathrm{H} 2}$ in dense gas as a function of local column density and metallicity, and allow star formation only from molecular gas. We also follow Hopkins et al. (2013) and restrict star formation to gas which is locally self-gravitating, i.e. has $\alpha \equiv \delta v^{2} \delta r / G m_{\text {gas }}(<\delta r)$ $<1$ on the smallest available scale ( $\delta r$ being our force softening or smoothing length). This forms stars at a rate $\dot{\rho}_{\star}=\rho_{\text {mol }} / t_{\mathrm{ff}}$ (i.e. 100 percent efficiency per free-fall time); so that the galaxy and even kpc-scale star formation efficiency is not set by hand, but regulated by feedback (typically at much lower values).

\footnotetext{
${ }^{4}$ Publicly available at http://galaxies.northwestern.edu/uvb

${ }^{5}$ The simulations get to a maximum density of $\sim 10^{4} \mathrm{~cm}^{-3}$
} 
Table 1. Simulations data. First column stands for the different parameters studied for each simulation. In columns $2-7$ results at $z=0$ for the simulations presented in this work are shown. Row (1): dark matter particle mass in the high-resolution region in $\mathrm{M}_{\odot}$. Row (2): fixed gravitational softening used for the dark matter particles in physical pc. Row (3): baryon particle mass in the high-resolution region in $\mathrm{M}_{\odot}$. Row (4): minimum baryonic force softening in pc (minimum SPH smoothing lengths are comparable or smaller). Recall that force softenings are adaptive (mass resolution is fixed). Row (5): virial mass in $\mathrm{M}_{\odot} \odot$ defined at the overdensity at which the spherical top hat model predicts virialization (Bryan \& Norman 1998). Row (6): maximum circular velocity in $\mathrm{km} \mathrm{s}^{-1}$. Row (7): virial radius in kpc. Row (8): halo spin (Bullock et al. 2001) definition. See Appendix A for more details. Row (9): halo concentration. See Appendix A for more details. Row (10): halo formation time. See Appendix A for more details. Row (11): virial baryon fraction, i.e. baryon mass inside the virial radius over the virial mass, divided by the cosmic baryon fraction. Row (12): virial gas fraction, i.e. gas mass inside the virial radius over the virial mass. Row (13): virial stellar fraction, i.e. stellar mass inside the virial radius over the virial mass. Row (14): stellar mass in $\mathrm{M}_{\odot}$. This is the stellar mass of the central galaxy. Row (15): effective stellar mass radius, i.e. half-stellar mass radius in kpc. Row (16): stellar iron over hydrogen ratio. Mass-weighted iron over hydrogen ratio for the dwarf stellar mass component. Row (17): total mass inside $500 \mathrm{pc}$ in $\mathrm{M}_{\odot}$. Row (18): dark matter mass inside 500 pc in $\mathrm{M}_{\odot}$. Row (19): baryon mass inside 500 pc in $\mathrm{M}_{\odot}$. Row (20): gas mass inside 500 pc in $\mathrm{M}_{\odot}$. Row (21): stellar mass inside 500 pc in $\mathrm{M}_{\odot}$.

\begin{tabular}{|c|c|c|c|c|c|c|}
\hline Parameter & $\begin{array}{l}\text { ULTRAFAINT_DM } \\
\text { (Collisionless) }\end{array}$ & $\begin{array}{c}\text { ULTRAFAINT }^{a} \\
\text { (Hydro: Feed-V) }\end{array}$ & $\begin{array}{c}\text { DWARF_DM } \\
\text { (Collisionless) }\end{array}$ & $\begin{array}{c}\text { DWARF_LATE } \\
\text { (Hydro: Feed-M) }\end{array}$ & $\begin{array}{c}\text { DWARF_MIDDLE } \\
\text { (Hydro: Feed-M-soft) }\end{array}$ & $\begin{array}{l}\text { DWARF_EARLY }{ }^{b} \\
\text { (Hydro: Feed-V) }\end{array}$ \\
\hline (1) $m_{\mathrm{p}}^{\mathrm{dm}}\left(\mathrm{M}_{\odot}\right)$ & $1.5 \times 10^{3}$ & $1.26 \times 10^{3}$ & $1.5 \times 10^{3}$ & $1.26 \times 10^{3}$ & $1.26 \times 10^{3}$ & $1.26 \times 10^{3}$ \\
\hline (3) $m_{\mathrm{p}}^{\text {bar }}\left(\mathrm{M}_{\odot}\right)$ & - & $2.54 \times 10^{2}$ & - & $2.54 \times 10^{2}$ & $2.54 \times 10^{2}$ & $2.54 \times 10^{2}$ \\
\hline (4) $\epsilon_{\mathrm{gas}}^{\mathrm{p}}(\mathrm{pc})$ & - & 1.0 & - & 2.0 & 25 & 2.8 \\
\hline (5) $M_{\text {vir }}\left(\mathrm{M}_{\odot}\right)$ & $3.2 \times 10^{9}$ & $2.5 \times 10^{9}$ & $9.2 \times 10^{9}$ & $7.6 \times 10^{9}$ & $7.7 \times 10^{9}$ & $7.7 \times 10^{9}$ \\
\hline (8) $\lambda$ & 0.031 & - & 0.0350 & - & - & - \\
\hline (9) $V_{\max } / V_{\mathrm{vir}}$ & 1.35 & - & 1.38 & - & - & - \\
\hline (10) $t_{50}(\mathrm{Gyr})$ & 1.43 & - & 1.84 & - & - & - \\
\hline (11) $f_{\mathrm{bar}}\left(\Omega_{\mathrm{m}} / \Omega_{\mathrm{b}}\right)$ & - & 0.024 & - & 0.093 & 0.074 & 0.056 \\
\hline (12) $f_{\text {gas }}$ & - & 0.0049 & - & 0.018 & 0.014 & 0.011 \\
\hline (13) $f_{*}$ & - & 0.00002 & - & 0.0004 & 0.0003 & 0.0003 \\
\hline$(18) M_{500}^{\text {dark }}\left(\mathrm{M}_{\odot}\right)$ & $1.6 \times 10^{7}$ & $1.7 \times 10^{7}$ & $2.6 \times 10^{7}$ & $0.43 \times 10^{7}$ & $0.80 \times 10^{7}$ & $1.6 \times 10^{7}$ \\
\hline (19) $M_{500}^{\mathrm{bar}}\left(\mathrm{M}_{\odot}\right)$ & - & $1.2 \times 10^{4}$ & - & $3.2 \times 10^{6}$ & $4.7 \times 10^{6}$ & $2.2 \times 10^{6}$ \\
\hline$(20) M_{500}^{\text {gas }}\left(M_{\odot}\right)$ & - & $6.9 \times 10^{2}$ & - & $3.1 \times 10^{6}$ & $4.5 \times 10^{6}$ & $1.6 \times 10^{6}$ \\
\hline$(21) M_{500}^{*}\left(\mathrm{M}_{\odot}\right)$ & - & $1.9 \times 10^{4}$ & - & $5.4 \times 10^{4}$ & $2.4 \times 10^{5}$ & $6.3 \times 10^{5}$ \\
\hline
\end{tabular}

Notes. ${ }^{a}$ Simulation presented in Hopkins et al. (2014a) as m09.

${ }^{b}$ Simulation presented in Hopkins et al. (2014a) as m10.

Feedback from stellar evolution is modelled by implementing energy, momentum, mass, and metal return from radiation, $\mathrm{SNe}$, stellar winds, and photoionization. Every star particle is treated as a single stellar population, with a known age, metallicity, and mass. Then all feedback quantities (the stellar luminosity, spectral shape, $\mathrm{SNe}$ rates, stellar wind mechanical luminosities, metal yields, etc.) are tabulated as a function of time directly from STARBURST99 stellar population synthesis model (Leitherer et al. 1999), assuming a Kroupa (2002) initial mass function (IMF). Details on the implementation of each of these physical processes in our simulations can be found in Hopkins et al. (2014a). No black hole physics has been considered in these simulations.

Despite taking all our inputs directly from stellar population models, there are some ambiguities in how we implement them. For example, when we deposit mass, momentum, and energy to particles within the SPH kernel, we can do so according to a massweighting or volume-weighting scheme. We have experimented with both, and we refer to these options as Feed-M and Feed-V, respectively.

We stress that the systematic differences due to these (and other similar) purely numerical choices (see appendix A of Hopkins et al. 2014a) are relatively small for integrated quantities like the stellar mass. However, since the dynamics of galaxies and star formation are chaotic, a small perturbation can make a non-negligible difference to the shape of the star formation history. These essentially stochastic variations will provide a useful means for us to examine the role of different star formation histories in shaping cores.

We have found that the main global parameters describing the dwarf galaxies are quite robust regardless of resolution, softening, and other minor changes in the code. See Appendix B for a full discussion on the convergence of our results.

\subsection{Sample summary}

A summary of all the relevant parameters used in the ultrahighresolution runs presented in this work is shown in Table 1 along with the naming conventions we have adopted. In this work we present a total of six high-resolution simulations of two dwarf galaxy haloes, one with a virial mass of $M_{\text {vir }}=3.2 \times 10^{9} \mathrm{M}_{\odot}$ and the other with $M_{\text {vir }}=9.21 \times 10^{9} \mathrm{M}_{\odot}$ (as measured in the high-resolution collisionless simulations). For the more massive halo we present here four runs, a high-resolution collisionless run (DWARF_DM) and a total of three different hydrodynamical runs which include two feedback implementation tests and the softening test mentioned above.

We have named the three hydrodynamical DWARF simulations based on their star formation histories (see Section 3.2 below). 

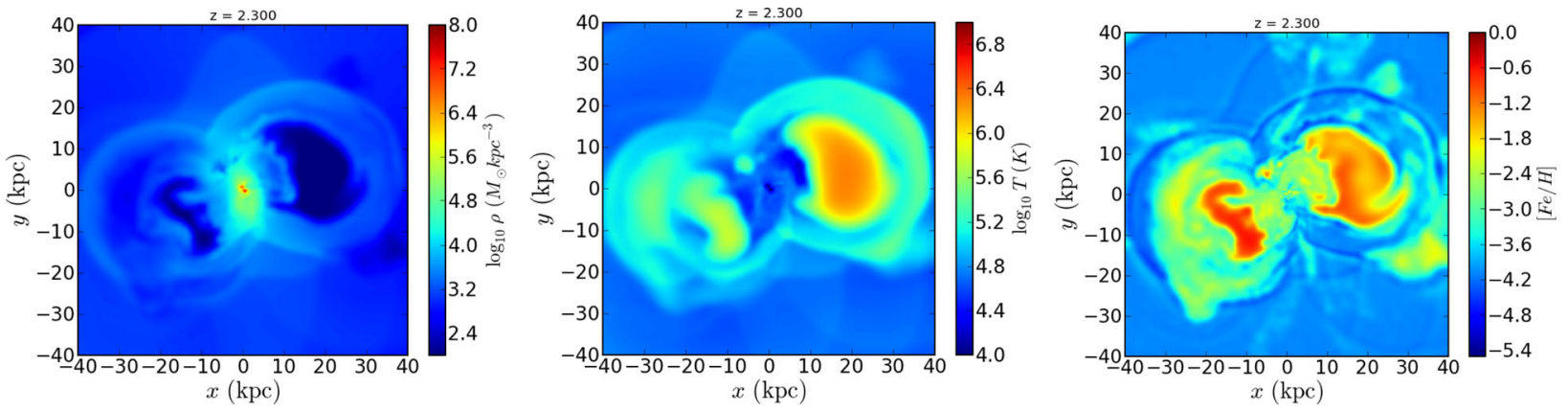

Figure 1. From left to right, visualizations of the gas density, gas temperature, and gas metallicity for the DWARF_EARLY run at $z=2.3$. All panels show the same thin slice along the $z$-axis centred at the main halo. The signatures of a recent stellar burst episode are clear in all of them.

The run we call 'DWARF_EARLY' shows most of its star formation at early times and corresponds with the feedback method Feed-V. The run we call 'DWARF_LATE' uses feedback method Feed-M and shows a more significant star formation rate (SFR) at low redshifts. The 'DWARF_MIDDLE' run is the softening test which uses feedback method 'Feed-M' and its SFR history stands just between the two. Simulations of the same dwarf using the 'Meshless Finite Mass' method implemented in Gizmo (Hopkins 2015) and the feedback Feed-V method produce results very similar to the 'DWARF_EARLY' run presented here (Fitts et al., in preparation).

For the smaller halo we have run the same number of simulations as we have for the larger one, but their results were so similar that we present only one hydrodynamic run (ULTRAFAINT, which uses Feed-V) and one collisionless run (ULTRAFAINT_DM). The hydrodynamical runs DWARF_EARLY and ULTRAFAINT were already presented in the first FIRE paper (Hopkins et al. 2014a).

As pointed out above, we have also checked the convergence of these results with resolution by running all these setups also at a lower resolution level. We discuss these runs in detail in Appendix $B$. We have also run many more $(\sim 50)$ simulations at this lower resolution level of these haloes to test other purely numerical issues and the effects of adding/removing each feedback mechanism in turn. Some of these are summarized in Hopkins et al. (2014a). We will not discuss them further in this paper because they are either not instructive for the study of this work because the included physics is not complete or because there is no change in the results. Even given excellent force and mass resolution, the time-step criterion used in simulations is always a concern if many, many orbits of $N$-body particles must be followed (as in the halo centres of the systems studied here). These can artificially deteriorate a central cusp, if an insufficiently stringent time-step criterion and/or error tolerance for the long-range force computations is used. We have therefore re-run a subset of our low-resolution runs, making the time-step criterion a factor of $\sim 30$, and force error tolerance a factor of $\sim 100$ times more strict than our default choices. This amounts to taking $<100 \mathrm{yr}$ time steps, with a tree force accuracy a factor of $\sim 1000$ stricter than used in Governato et al. (2012), and a factor of $\sim 100$ stricter than was found to give good convergence in idealized comparisons of dark matter zoom-in simulations in Kim et al. (2014). Given our very strict default tolerances, this gave well-converged results.

Fig. 1 shows visualizations of the gas density (left-hand panel), gas temperature (middle panel), and gas metallicity (right-hand panel) for the DWARF_EARLY run at $z=2.3$. All panels show the same thin slice along the $z$-axis centred at the main halo. The signatures of a recent $\mathrm{SN}$ episode are clear in all of them.

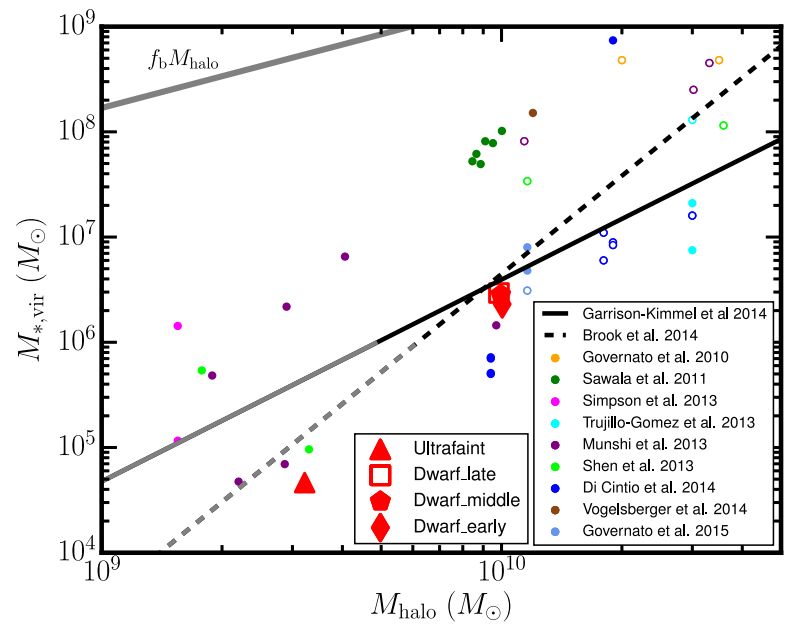

Figure 2. Galaxy stellar mass-halo mass relation at $z=0$ for the simulations presented in this work. Filled red triangle stands for the ULTRAFAINT run. Empty red square, filled red pentagon, and filled red diamond stand for the DWARF_EARLY, DWARF_MIDDLE, and DWARF_LATE runs, respectively. Circles stand for other published simulations in the literature using subgrid stellar feedback models. Empty symbols stand for runs in which a dark matter core was found according to the typical definition used in previous works: a slope $a \gtrsim-0.9$ for the dark matter density profile between 1 and 2 per cent of the virial radius. The two black lines show abundance matching relations derived by Brook et al. (2014) and Garrison-Kimmel et al. (2014a) using galaxy counts within the Local Volume. These relations should be complete to $M_{\star} \sim 10^{6} \mathrm{M}_{\odot}$ (much deeper than other published abundance matching relations and therefore the most relevant for this comparison). To make all simulation data and abundance matching results consistent between each other, we have plotted the total virial masses of the dark-matter-only runs defined as $\Delta_{\text {vir }}=200 \rho_{\text {crit }}$. A small correction has been applied whenever these values were not available in the literature (with $\leq 10$ per cent effects). In order to facilitate direct comparison with other results in the literature, the stellar mass plotted is all of the stellar mass inside the virial radius. Using a smaller radius makes only a small difference for our simulated dwarf galaxies.

\section{RESULTS}

\subsection{Basic properties at $z=0$}

Table 1 presents some relevant parameters describing the properties of each simulation presented in this work that will allow an immediate comparison with previous simulations and observations of dwarf galaxies.

Of particular interest is the resultant stellar mass in each dwarf. Fig. 2 presents the stellar mass-halo mass relation for the four 

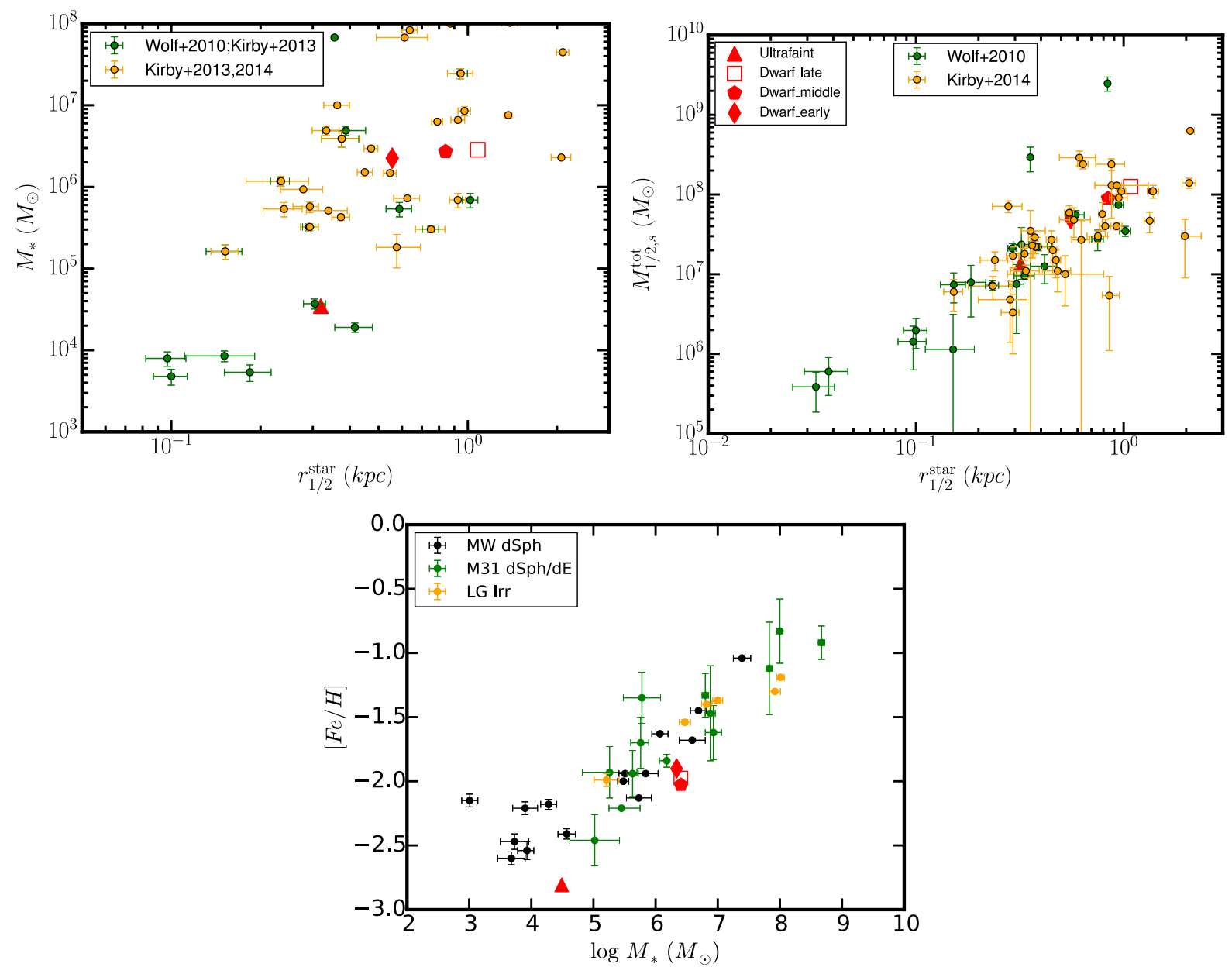

Figure 3. All panels show the hydrodynamical runs presented in this work compared with different observations at $z=0$. Empty red square, filled red pentagon, and filled red diamond stand for the DWARF_EARLY, DWARF_MIDDLE, and DWARF_LATE runs, respectively. Upper left-hand panel: stellar mass versus effective stellar mass radius. Circles stand for observational data from Wolf et al. (2010) and Kirby et al. (2013, 2014). Upper right-hand panel: the galaxy size (effective stellar mass radius) versus mass (total mass inside this radius) relation for the simulations presented in this work. In order to compare it with observations, we have plotted the data derived by Wolf et al. (2010) (green circles) and Kirby et al. (2014) (orange circles). Bottom panel: stellar mass-stellar metallicity relation for the simulations presented in this work. Circles stand for the data compiled by Kirby et al. (2013) for nearby dwarfs. See text for more details.

hydrodynamical runs described above (large red points) compared to the most recent estimates for this relation from abundancematching exercises in the Local Group (Brook et al. 2014; GarrisonKimmel et al. 2014a, black dashed and solid lines, respectively). The known sources of stellar feedback we include, with no adjustment, automatically produces galaxy stellar masses that are consistent with those required to match local galaxy counts. ${ }^{6}$ For the halo mass range presented here, this is particularly impressive, as the integrated stellar mass is suppressed by factors of $\sim 1000$ relative to the universal baryon fraction (upper solid grey line).

The smaller points in Fig. 2 show results from previous hydrodynamical simulations of dwarf galaxies (Governato et al. 2010; Sawala et al. 2011; Munshi et al. 2013; Simpson et al. 2013; Shen et al. 2014; Trujillo-Gomez et al. 2015). The open points are those that have reported at least mild flattening of the central dark matter cusp in response to feedback effects. We note that all of those open

\footnotetext{
${ }^{6}$ Abundance matching results below $\sim 10^{6} \mathrm{M}_{\odot}$ stellar mass are extrapolation as observations throughout the Local Group are not complete below this limit.
}

points are associated with systems that have formed a fair number of stars, with $M \star \gtrsim 7 \times 10^{6} \mathrm{M}_{\odot}-$ more massive than the systems of concern for the 'too big to fail' problem. As we discuss below, one of our runs (DWARF_LATE, open square) produces a large core while forming significantly fewer stars.

The upper left-hand panel of Fig. 3 shows galaxy size, measured as the half-stellar mass radius versus the total stellar mass of the galaxy for our simulated galaxies (red points). The observed stellar size-mass relation seen for Milky Way satellites (green) and Local Group field dwarfs (yellow) are shown as data points (taken from Wolf et al. 2010; Kirby et al. 2013, 2014, who also compile data from the literature). The upper right-hand panel of Fig. 3 shows the total mass within the half-stellar mass radius versus the halfstellar mass radius, again for our simulated galaxies compared to local galaxies. ${ }^{7}$ Finally, the bottom panel shows the stellar massstellar metallicity relation. Overall, the simulated galaxies are in good agreement with sizes, metallicities, and total masses seen for

\footnotetext{
${ }^{7}$ When we do this comparison with observations we are assuming that the half-stellar mass radius is equivalent to the half-light radius.
} 
galaxies of their stellar mass in the local Universe. For example, the DWARF_LATE and middle runs show a good agreement with Fornax. DWARF_EARLY's size and mass are close to Ursa Minor.

Table 1 shows that DWARF_EARLY is much more dark matter dominated within $500 \mathrm{pc}$ than DWARF_LATE. This also holds when we look at the half-stellar mass radius of each galaxy, instead of at a fixed physical value. Within this radius, DWARF_EARLY has $M_{\text {tot }} / M_{\star} \approx$ 44 and $M_{\text {tot }} / M_{\text {baryons }} \approx 11$. By mass, stars are subdominant to gas within the half-light radius by a factor of 2.8 for this dwarf. Within the half-mass stellar radius, DWARF_LATE has $M_{\mathrm{tot}} / M_{\star} \approx 87$ and $M_{\text {tot }} / M_{\text {baryons }} \approx 5.4$ owing to a large reservoir of gas within the stellar half-mass radius $\left(M_{\mathrm{gas}}=16 M_{\star}\right.$ within $\left.r_{1 / 2}^{*}\right)$.

\subsection{Star formation histories}

While the simulated DWARFS (early, middle, and late) all show simi$\operatorname{lar} z=0$ stellar masses, they arrived at those final states via different paths. The ULTRAFAINT run, on the other hand, ends up with a stellar mass some two orders of magnitude smaller than any of the DWARF runs, though it resides within a halo that is only $\sim 3$ times less massive. In this subsection, we explore these differences by examining the star formation histories in some detail.

In Fig. 4, we present the SFRs (left-hand panel) and the normalized cumulative star formation histories (right-hand panel) of all four high-resolution hydro runs. The ULTRAFAINT simulation (orange line) forms all of its stars before $z \sim 2.5$. The galaxy shuts down at this redshift because of two main effects: (1) the ultraviolet (UV) background prevents fresh gas accretion after $z \sim 6$ and (2) stellar feedback acts to self-quench the system after the ionizing background turns on. The remaining cold gas found at $z=0$ is not able to reach high enough densities to generate stars. Also notice that even though the UV background starts acting at high redshift on the gas particles, there is still active star formation for about one billion years after this redshift.

This is not unexpected: previous lower resolution simulations predicted that reionization-induced UV heating is not strong enough to remove all of the gas from dwarf-sized haloes (Hoeft et al. 2006). However, these simulations were not able to resolve the star formation histories of these galaxies, so it was not clear if the remaining gas would be able to form stars. As the UV background effectiveness depends on the density of the gas, cold and dense gas is not affected. Moreover, this more efficient star formation period seems crucial in order to match the stellar metallicity ratios observed for low-mass dwarf galaxies (Kirby et al. 2013).

Fig. 4 also shows the SFRs of the three DWARF runs. DWARF_EARLY forms more than half of its stars prior to $z=2.5$ while DWARF_LATE maintains a fairly substantial SFR down to $z=0$. The DWARF_MIDDLE SFR history stands just between the two. All of the runs show bursty star formation histories on $\sim 100 \mathrm{Myr}$ time-scales.

In all three of the DWARF runs, the star formation histories show two different phases. At the highest redshifts $(z>3)$, total dark halo and stellar masses both grow efficiently (albeit with some offset). This is the 'rapid assembly' phase (Wechsler et al. 2002), before/during reionization, in which feedback, while able to eject some gas from the galaxy and provide some overall suppression and variability of the star formation, does not appear to dominate the gas dynamics (the central potential and mass of the halo grow on time-scales comparable to the galaxy dynamical time). But from $z \sim 3$ onward, halo accretion rates slow down and feedback acts strongly. From this point on, there appears to be a steady-state SFR that can be considered constant with time when averaged over a Gyr scale (a bursty behaviour emerges when smaller time bins are used). In this phase, the galaxy is able to cycle new material into a fountain and so maintain equilibrium. This 'quasi-equilibrium' SFR scales with the central potential of the galaxy (see Hopkins et al. 2012), as traced by quantities such as the central halo density or $V_{\max }$ (the maximum circular velocity), not the halo mass or virial velocity. The central potential depth increases only weakly over this time as the halo accretes material mostly on its outskirts. This low but constant SFR at low redshift is a key factor in shaping the final matter structure of the dwarf galaxy and will be discussed in detail in the next section.

Observations of the SFR of dwarf galaxies (Tolstoy, Hill \& Tosi 2009; Brown et al. 2014; Cole et al. 2014; Skillman et al. 2014; Weisz et al. 2014) show a relatively high dispersion for a fixed stellar mass, but all the histories of our simulated galaxies seem realistic when compared with these data. In particular, our ULTRAFAINT galaxy is composed of uniformly old stars, as observed in real ultrafaint
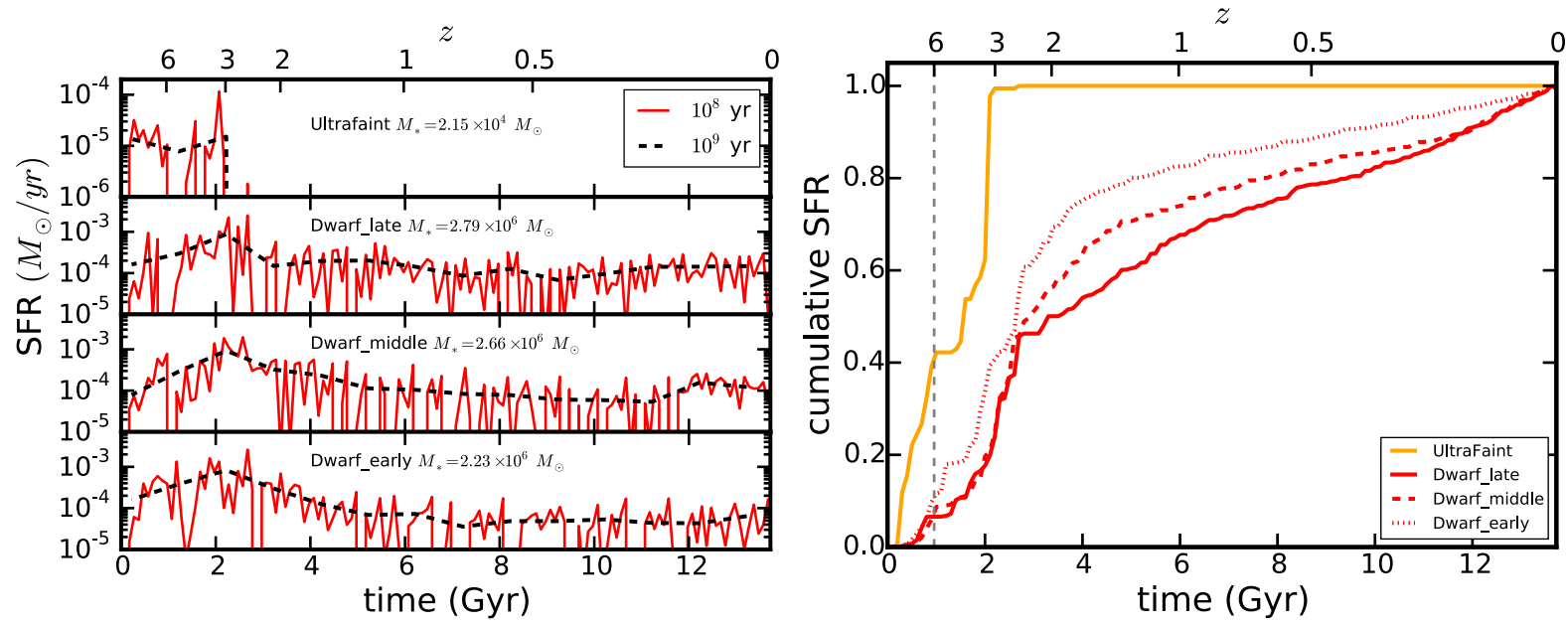

Figure 4. Left-hand panel: star formation rates for the hydrodynamical runs presented in this work obtained using two different time bins: $10^{8}$ yr (full red lines) and $10^{9} \mathrm{yr}$ (dashed black lines). Right-hand panel: normalized cumulative SFR history for all the hydrodynamical runs presented in this work. Notice the difference of the star formation histories among the three high-resolution runs. The vertical grey dashed line marks the reionization redshift assumed in the simulation. Simulated star formation histories are very similar to some observed ones (e.g. Skillman et al. 2014; Weisz et al. 2014). See text for more details. 


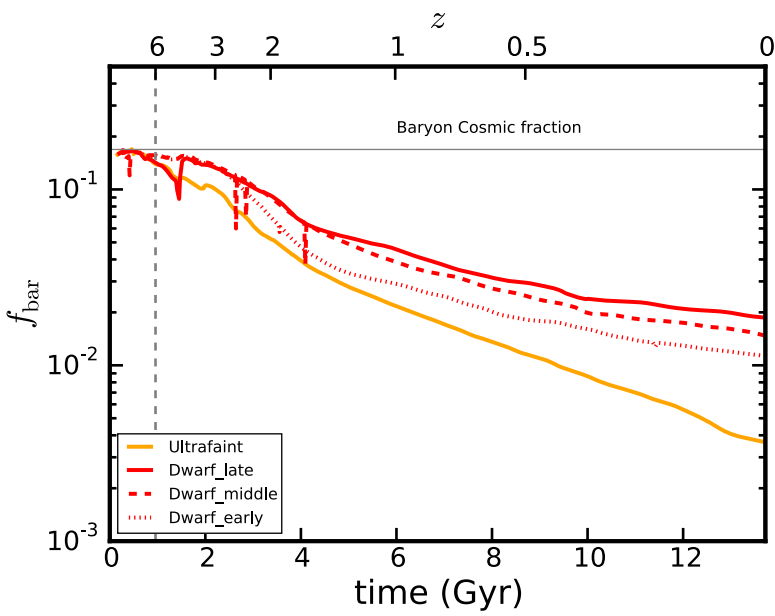

Figure 5. Evolution of the virial baryon fraction for all the hydrodynamical runs presented in this work. In all cases, gas is slowly expelled out of the halo by the stellar feedback. Notice how the DWARF runs with the highest SFR at early times (when the halo is still growing) end up having a lower baryon fraction. The ULTRAFAINT is further affected by the UV background, which prevents accretion of gas after $z \sim 6$.

dwarf satellites of the Milky Way (Brown et al. 2014), perhaps making them fossils of reionization (Ricotti \& Gnedin 2005; Bovill \& Ricotti 2011).

Some insight into the extremely low efficiency of star formation in all of these systems can be gained from examining the total baryon fraction versus time within their associated virial radii. This is shown in Fig. 5. Specifically, the virial baryon fraction (baryon mass divided by total mass inside the virial radius) begins declining in the ULTRAFAINT run from the moment the UV background starts acting to reduce the amount of gas falling into the halo. This allows feedback to be more efficient in expelling the gas out of the halo potential. The DWARF runs also begin to demonstrate a steady decline in their baryon fractions, but only after $z \sim 2.5$. This is the redshift when the halo itself stops growing (the end of the 'rapid accretion phase'). Below that redshift, star formation feedback steadily acts to expel gas. The runs with the higher SFRs have a lower baryon fraction, though all three of the DWARF runs end up at a factor of $\sim 10-20$ below the cosmic mean (horizontal grey line). However, the stellar feedback in the DWARF_EARLY simulation has managed to expel a larger fraction of material from halo, slowing down late time star formation.

One intriguing result from Fig. 5 is that the overall baryon fraction decreases steadily, without global jumps that are tightly linked to the SFR (which varies substantially over $\sim 100$ Myr time-scales). Instead, the baryons slowly 'evaporate' out. This is in contrast with other studies (Sawala et al. 2011; Simpson et al. 2013) that show sharper jumps. However, the lower resolution used by Sawala et al. (2011) could explain why their evolution is less smooth. Simpson et al. (2013) used comparable resolution to this work but studied a lower mass system, $10^{9} \mathrm{M}_{\odot}$, which could explain the much more drastic effect due to the UV background in the gas virial fraction that they found.

In the next section, we study how these differences in the star formation histories affect the matter distribution of the halo.

\section{DARK MATTER CONTENT AND STRUCTURE}

In Fig. 6, we present the dark matter density profiles of the hydrodynamical ULTRAFAINT (left) and DWARF (right) runs compared with their equivalent collisionless run at $z=0$. The grey bands mark the regions where the simulations are not fully converged according to the criterion of Power et al. (2003) computed for the dark-matteronly simulation. The Power et al. radius is defined to be the radius where the two-body relaxation time, $t_{\text {relax }}$, becomes shorter than the age of the Universe $t_{0}$, where $t_{\text {relax }}$ is determined by the number of particles and the average density of the enclosed region $\bar{\rho}$. Specifically, Power et al. found that $t_{\text {relax }}<0.6 t_{0}$ is the best criterion. Elbert et al. (2015) have recently confirmed that this criterion is accurate using zoom simulations of collisionless dwarf haloes at similar resolution to those we examine here. The vertical black dotted lines in Fig. 6 mark four times the dark matter gravitational softening used in the collisionless runs. We note that while radii larger than the Power et al. radius should not suffer from two-body relaxation,
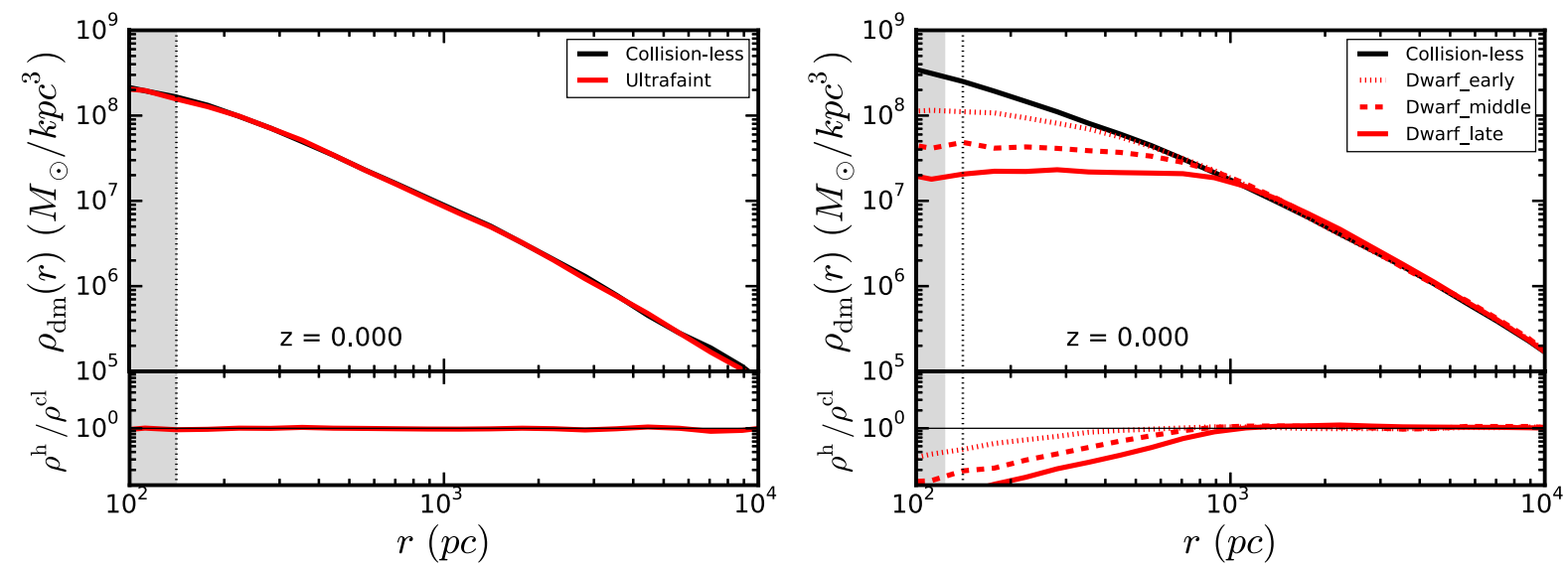

Figure 6. Left: the dark matter density profile at $z=0$ for the collisionless (black line) and hydrodynamical (red line) runs of the $3 \times 10^{9} \mathrm{M}_{\odot}$ halo. The 'collisionless' line has been converted to the effective dark matter density by accounting for the fact that a fraction $\Omega_{\mathrm{b}} / \Omega_{\mathrm{m}}$ of each particle is assumed to be baryonic in these runs. The bottom panel shows the ratio between the two profiles. Right: the same for the DWARF halo runs, where each hydrodynamical run is marked by a different style of red line. Grey shaded area marks the region below the convergence radius defined using Power et al. (2003) criteria for the collisionless run. The vertical black dotted line marks four times the dark matter gravitational softening used in the collisionless runs. Note that the DWARF_LATE run has produced a large $(\sim 1 \mathrm{kpc})$ constant-density core, while the DWARF_EARLY has a dark matter profile that is very similar to the dissipationless simulation for radii that are well converged. The dark matter in the hydrodynamic uLTRAFAINT run is identical to that of the dissipationless case. 

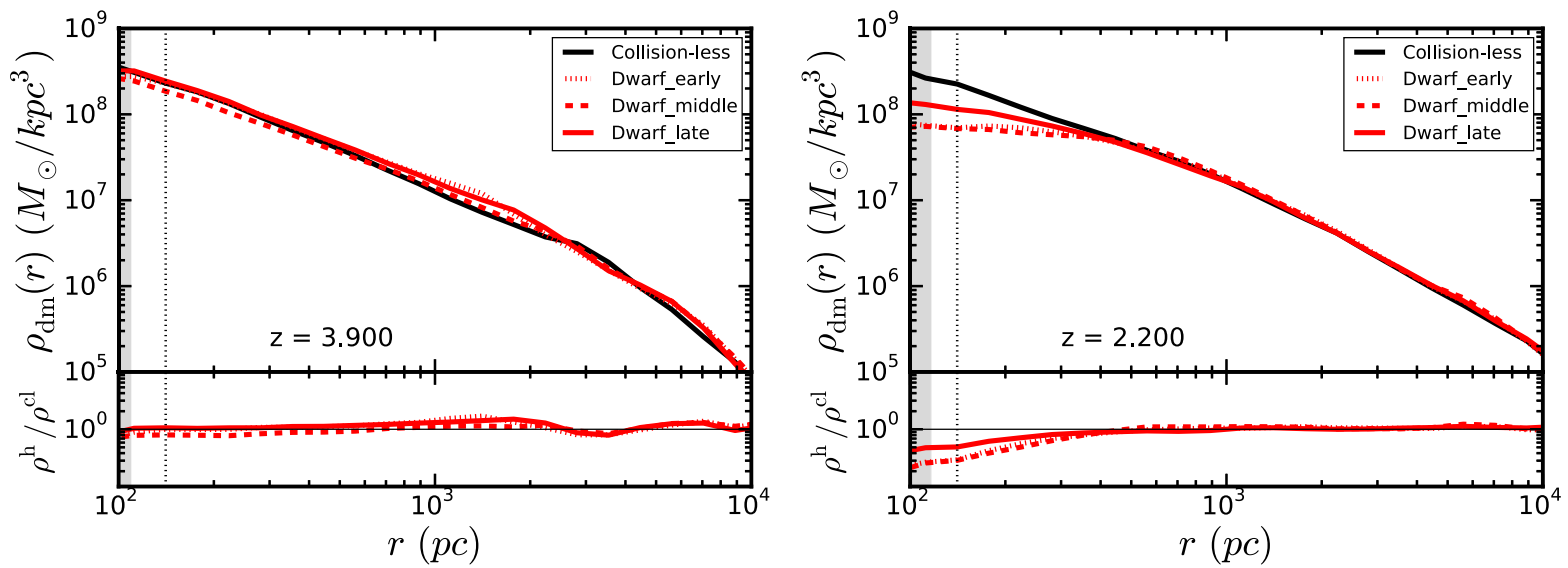

Figure 7. Time variation in density profiles. The dark matter density profile at $z=3.9$ (left-hand figure) and $z=2.2$ (right-hand figure) for the collisionless (black) and hydrodynamical (red lines) runs of the $1 \times 10^{10} \mathrm{M}_{\odot}$ halo. Bottom panel shows the ratio between the two profiles. The vertical black dotted line marks four times the dark matter gravitational softening used in the collisionless runs. Grey shaded area marks the region below the convergence radius defined using Power et al. (2003) criteria for the collisionless run. Note that, at $z=2.2$, DWARF_LATE has a higher central density than DWARF_EARLY. Late-time star formation in DWARF_LATE serves to reduce the dark matter halo's density in the centre by a factor of $\sim 5$ by $z=0$ (see right-hand panel of Fig. 6 ), while DWARF_EARLY has little star formation subsequent to $z=2.2$. Its density profile remains essentially unchanged from $z=2.2$ to 0 .

the smallest radius where results in hydrodynamical simulations are converged may be (significantly) larger.

The left-hand panel of Fig. 6 shows results for the ULTRAFAINT simulations. In this case, there is no sign of a decrease in the dark matter density in the hydrodynamical run; in fact the dark matter profile matches perfectly with the collisionless run. In the DWARF runs (right-hand panel), we observe that all hydrodynamical runs show varying levels of decrease of the inner dark matter density when compared with their equivalent collisionless run. In particular, the DWARF_LATE run has produced a fairly large $(\sim 1 \mathrm{kpc})$ constantdensity core - this is exactly the behaviour needed to help alleviate the 'too big to fail' problem (see e.g. Elbert et al. 2015; Governato et al. 2015) and that would be required to explain indications of cored profiles in low-mass galaxies in the Local Group (Donato et al. 2009; Walker \& Peñarrubia 2011; Salucci et al. 2012; Amorisco et al. 2014; Burkert 2015).

One common way to quantify core formation in haloes is to measure the log-slope of the density profile $\alpha$ at $1-2$ percent of the virial radius. The DWARF halo in the dark-matter-only run has $\alpha=-1.58$, while DWARF_EARLY, DWARF_MIDDLE, and DWARF_LATE have $\alpha=-1.39,-0.88$, and -0.27 , respectively. The late-forming dwarf produces the shallowest profile and the largest core, while the early-forming dwarf produces the densest, cuspiest system.

Over time, in all three dwarf runs, we have observed clear correlations between core formation and star formation events. However, at early times, as the haloes continue to accrete matter and experience central mergers, the cusps regrow regularly. During the early, rapid accretion phase, evolution in the density structure is fairly stochastic, with cores forming in response to blowout events, and then becoming erased as cusps reform in response to mergers. Fig. 7 illustrates the formation of the dark matter core using two time steps. Shown are the dark matter density profiles of the DWARF runs at $z=$ 3.9 (left) and $z=2.2$ (right). At $z=3.9$, very little star formation has occurred and the halo is experiencing very rapid growth and we see no decrease in core dark matter density compared to the collisionless run. However, at $z=2.2$, there are some signs of a decrease in the central dark matter density in the hydro runs. Interestingly, DWARF_LATE - which has the largest core at $z=0$ - has the smallest core profile at $z=2.2$. DWARF_EARLY shows almost the opposite trend, owing to the fact that it has had more star formation by $z=2.2$ than the later forming dwarf.

To further explore the evolution of the dark matter density with time, Fig. 8 compares the cumulative star formation history (dashed curves, normalized to unity at the present day) and the mass interior to radii of $0.3,0.75$, and $2 \mathrm{kpc}$ relative to the collisionless run as a function of time for DWARF_LATE (left-hand panel) and DWARF_EARLY (right-hand panel). In both cases, the early phases of galaxy formation $(z \gtrsim 3)$ result in fluctuations in the inner mass profiles of these galaxies (Davis, Khochfar \& Dalla Vecchia 2014). After $z=3$, when the dark matter assembly of each halo is essentially complete, DWARF_EARLY forms only a relatively small amount of stars. This results in at most a slight reduction in the inner dark matter mass (right-hand panel). DWARF_LATE, however, forms more than 50 per cent of its stellar mass after $z=2$. Most of the density reduction also occurs after this phase, pointing to a link between the final densities of these objects and their late-time star formation histories. This is consistent with Laporte \& Peñarrubia (2015), who found that cusps can regrow after early core formation.

Fig. 9 further illustrates the correlation between star formation history and core formation, now with the early and late runs on the same plot, and using dimensional star formation histories rather than normalized ones. Specifically, the cumulative star formation histories of the DWARF_EARLY (green dash) and DWARF_LATE (red dash) runs are shown along with the evolution of the ratio of dark matter enclosed within $0.3 \mathrm{kpc}$ for the hydrodynamic compared to the dark-matter-only runs (solid lines). It is clear that a higher SFR at late times (from $z \sim 2$ ) produces a bigger decrease in the central dark matter density. Notice that although this difference in the SFR below $z=2$ produces very different cores, the difference in the total amount of stars at $z=0$ is minimal (see Fig. 4 and Table 1). In concordance with this picture, lower resolution runs, which have slightly higher SFRs at low redshift than their high resolution counterparts, show bigger cores in their dark matter distribution (see Appendix B).

It is likely that the relationship between when the stars form and core formation is most important at this critical stellar mass/halo mass scale $\left(M_{*} \sim 2-3 \times 10^{6} \mathrm{M}_{\odot}\right.$ within $\sim 10^{10} \mathrm{M}_{\odot}$ haloes $)$. Previous simulation efforts (Pontzen \& Governato 2012; Di Cintio et al. 

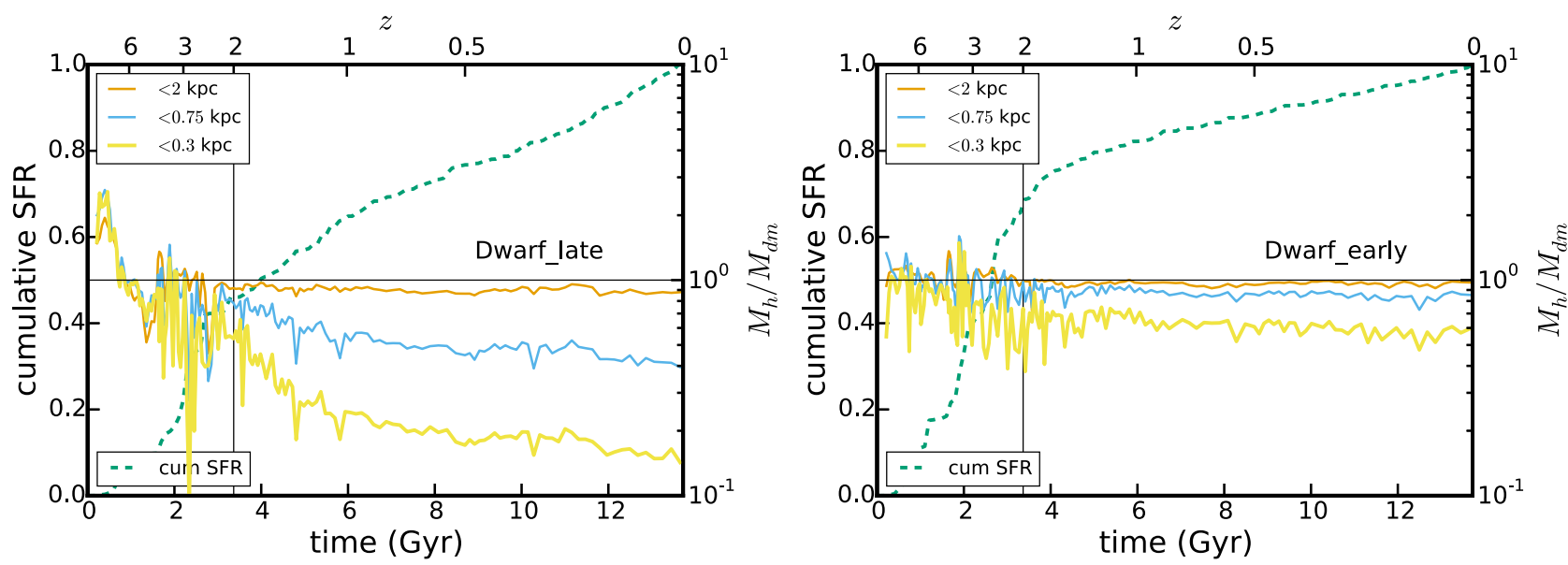

Figure 8. Correlation between star formation history and core formation for DWARF_LATE (left) and DWARF_EARLY (right). The green dashed lines are the cumulative star formation histories for each run normalized to its value at $z=0$. The vertical line indicates the end phase of the rapid-accretion epoch feedback after this time is most capable of forming stable cores that are not regrown by subsequent mergers. The solid lines show the integrated mass within different radii (as labelled) divided by the same mass in the dissipationless runs - values less than unity indicate the formation of a lower density core than in the dark-matter-only case. We see that in the DWARF_LATE case (left), the core begins to form after the rapid accretion phase has ended $(z \sim 2)$ and keeps growing slowly down to redshift $z=0$ as star formation continues. DWARF_EARLY, however, forms most of its stars prior to the quiescent accretion phase and a large core never takes hold.

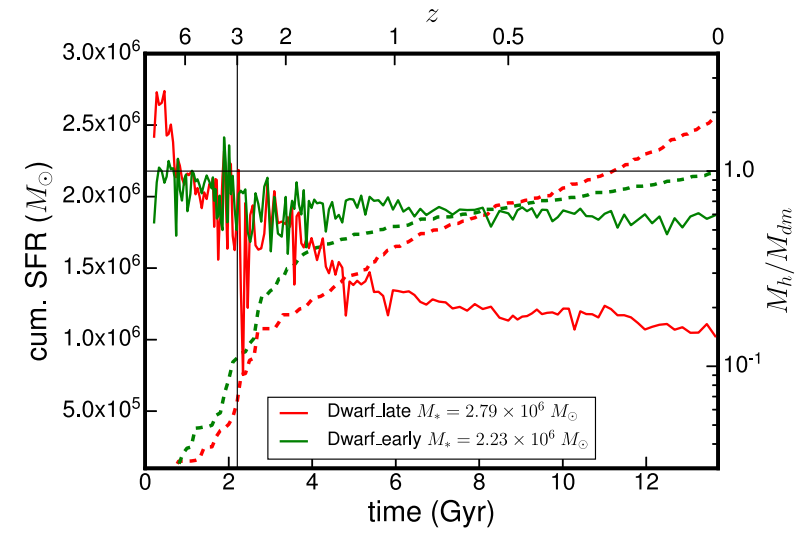

Figure 9. The cumulative star formation history (dashed lines) and the dark matter mass ratio between the hydrodynamical run and the collisionless run at $0.3 \mathrm{kpc}$ (full lines) for the early and late forming DWARF runs.

2014) have found that dark matter cores are usually not created in galaxies with so few stars in haloes below $\sim 10^{10} \mathrm{M}_{\odot}$. We suggest that at this critical mass scale, where the energy from feedback sources is just at the edge of that required for core formation, small variations in star formation histories can significantly alter the result. Indeed, in our general analysis of the dark matter properties in all FIRE runs, we find a similar transition around $10^{10} \mathrm{M}_{\odot}$ (Chan et al., 2015).

\subsection{Energy considerations}

Recently, there has been some discussion in the literature about the energy requirements for the formation of a core in a dwarf galaxy halo (see e.g. Peñarrubia et al. 2012; Garrison-Kimmel et al. 2013; Teyssier et al. 2013, and references therein) - specifically, how many stars are required for there to be enough energy available to create a core? At first comparison, the fact that the DWARF_LATE simulation was able to produce a sizable core with so few stars appears to be in contradiction to the results of Garrison-Kimmel et al. (2013), who suggested that cores this large are not energetically possible. However, the host halo considered in Garrison-Kimmel et al. (2013) is more concentrated than the one we consider here. In order to explicitly check whether our results make sense energetically we aim to compare the energy released in $\mathrm{SNe}$ in our simulations to the difference in dark matter gravitational energy potential of the dwarf hydro runs and its collisionless version.

The gravitational potential energy is defined as

$U=-4 \pi G \int_{r_{\min }}^{r_{\max }} r M(r) \rho(r) \mathrm{d} r$.

We computed this value numerically directly from the simulation data. We considered $r_{\text {min }}=0$ (using $r_{\min }=\epsilon_{\mathrm{dm}}$ or $r_{\min }=\epsilon_{\mathrm{gas}}^{\min }$ gives very similar results) and $r_{\max }=2 \mathrm{kpc}$. We used this maximum radius because we are interested in the energy necessary to decrease the inner part of the density profile and from this point the dark matter profiles match almost exactly (see Fig. 6). More importantly, at larger radii differences between the gravitational energy potential can be significant just due to the exact position of the substructure between the collisionless and the hydrodynamic runs. Therefore this definition of potential energy for each run sets a lower energy limit on the amount of energy necessary to create a specific dark matter decrease in the inner part of a halo. We define $\Delta U_{\mathrm{dm}}$ as the difference between the potential energy of the hydrodynamical run and the potential energy of the collisionless run.

In order to obtain an estimate of the energy available from feedback we have considered the energy available from SNe using the parameters from our simulations: $E_{\mathrm{tot}}=\left(M_{\mathrm{star}} / m\right) f E_{\mathrm{sn}}$, where $E_{\mathrm{sn}}=1 \times 10^{51} \mathrm{erg}$ is the energy of one $\mathrm{SN}, f=0.0037$ is the fraction of stars more massive than $8 \mathrm{M}_{\odot}$ for a Kroupa (2002) IMF, and $m$ $=0.4 \mathrm{M}_{\odot}$ is the mean stellar mass. The stellar mass of the central galaxy at $z=0$ is in the range $\sim 2.3-2.8 \times 10^{6} \mathrm{M}_{\odot}$; however, from Fig. 8 we can see that the core starts to form below $z \sim 2$, therefore, we have also considered the stellar mass produced since this time until $z=0$. We are considering just SN energy but in principle, just taking into account the energy, the contribution from photoionization and radiation pressure could play a role in core creation. Although most of the energy in radiation just escapes the system, 


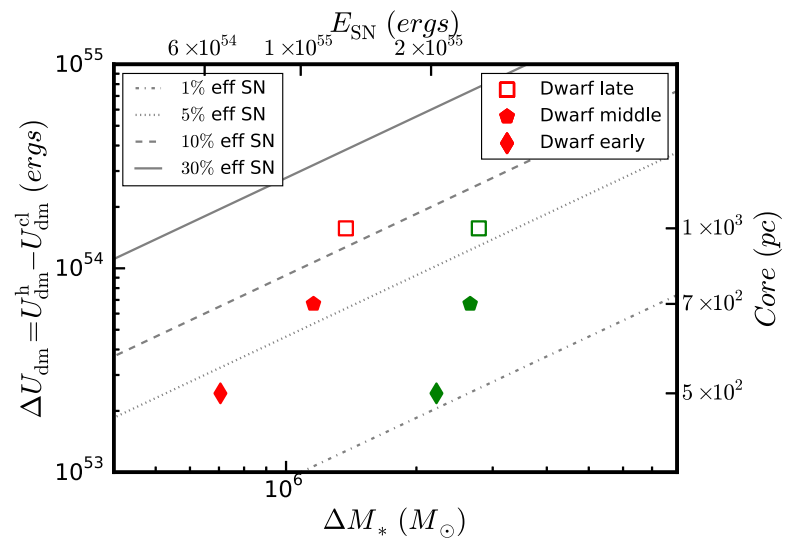

Figure 10. Energy considerations in the formation of the dark matter cores in a $10^{10} \mathrm{M}_{\odot}$ halo. The $x$-axis shows the total stellar mass for each of the dwarf runs (green symbols) and the amount of stellar mass formed between redshift $z=2$ and 0 (red symbols). The upper axis shows the energy expelled by $\mathrm{SN}$ from this stellar population. The $y$-axis shows the difference in potential energy between the hydrodynamical run and the collisionless run. The right $y$-axis shows the size of the core created due to this difference of energy. See text for details.

there is in principle $\sim 100$ times more energy in radiation than in SNe. Preliminary tests done in this regard by changing the energy per $\mathrm{SN}$ do not point towards a relevant role of these processes, at least as they are currently implemented in the code. However, we leave a more careful analysis to future work.

In Fig. 10, we plot the potential energy difference between the hydrodynamical runs and the collisionless run, $\Delta U_{\mathrm{dm}}$, versus the total stellar mass (green symbols) and the stellar mass produced since $z=2$ down to $z=0$ (red points) for all the different DWARF runs. The SN energy available from the stellar mass and the size of the core ${ }^{8}$ linked with the difference in energy are also marked in the figure. Grey lines stand for different efficiencies of the $\mathrm{SN}$ $(1,5,10$, and 30 percent), i.e. the energy from SN affecting the dark matter. Our simulations indicate that the gravitational potential energy of the halo has changed by an amount consistent with $\sim 5-10$ per cent of the SNe energy available for the DWARF_LATE run, while only $\sim 1$ per cent of the total SNe energy has been effectively captured by the dark matter in the DWARF_EARLY run, owing to the fact that many of these $\mathrm{SNe}$ exploded during the period of rapid accretion, when cusps were reforming. We find similar results when considering the lower resolution runs, including efficiencies, as they have slightly bigger cores than their counterparts but a higher SFRs at lower redshift (see Appendix B). Overall, these results suggest that large cores induced by star formation feedback will never appear in galaxies smaller than $\sim 10^{5} \mathrm{M}_{\odot}$ (in $\sim 2-3 \times 10^{9} \mathrm{M}_{\odot}$ or bigger haloes), owing to the inefficiency of $\mathrm{SNe}$ energy coupling to the dark matter.

\section{SUMMARY AND CONCLUSIONS}

We have performed several high-resolution zoom-in hydrodynamical simulations of an ultrafaint galaxy halo $\left(3 \times 10^{9} \mathrm{M}_{\odot}\right)$ and a dwarf galaxy halo $\left(1 \times 10^{10} \mathrm{M}_{\odot}\right)$. Our simulations include all major sources of stellar feedback, implemented directly from stellar evolution calculations. Without parameter tuning, the code reproduces

\footnotetext{
${ }^{8}$ We define the size of the core at the radius where the mass ratio between the hydrodynamical over the collisionless runs is 0.9 (see Fig. 6).
}

a relation between galaxy stellar mass and halo mass that is consistent with observations. Moreover, we find that global properties of these simulated haloes - including their characteristic sizes, metallicities, and gas contents - are well matched to observed galaxies of similar stellar mass. These global properties describing the simulated dwarfs are robust to changes in force and mass resolution. Furthermore, the feedback models and the outflows they generate are inherently multiphase, matching observations. The predictive nature of our galaxy formation model is particularly important, as the model does not contain ad hoc numerical solutions adopted by other models, e.g. cooling shut-offs or prescribed wind properties, that contain adjustable parameters. The mass scale of our simulated dwarfs $-M_{\star} \sim 2 \times 10^{6} \mathrm{M}_{\odot}-$ is particularly relevant because previous models able to generate cores have usually formed almost an order of magnitude more stars in such haloes $\left(M_{\mathrm{vir}}=10^{10} \mathrm{M}_{\odot}\right)$. Such galaxies are too massive in terms of the number of stars given their halo mass and therefore cannot be typical, given observed galaxy counts around the Milky Way and generic predictions from $\Lambda \mathrm{CDM}$ simulations (Brook et al. 2014; Garrison-Kimmel et al. 2014b).

Our models show a slow but continuous decrease of the baryonic mass inside the virial radius after $z \sim 6$. The UV background, in concert with star formation feedback, plays a fundamental role in regulating star formation in low-mass systems and appears to be the driving factor in suppressing gas accretion in our ultrafaint run. However, for the halo masses studied in this work, the UV background does not shut down star formation immediately because it is not efficient in heating the high-density gas in the centre of these haloes. The simulated ultrafaint $\left(M_{\mathrm{vir}}=3 \times 10^{9} \mathrm{M}_{\odot}\right)$ continues forming stars for $\sim 2 \mathrm{Gyr}$ following reionization (at which time it runs out of cold gas; such an object would be a counterpart in the field to known ultrafaint satellites of the Milky Way), while the more massive dwarfs continue to form stars to $z=0$. This may indicate a transition from lower mass objects that are incapable of acquiring cold gas after reionization to dwarfs at this mass scale that can continue to accrete fuel for subsequent star formation.

We have also studied, in detail, the dark matter distribution of these haloes. The simulated dwarfs $\left(M_{\star} \sim 2 \times 10^{6} \mathrm{M}_{\odot}\right)$ have a variety of density profiles, ranging from a small modification of the equivalent dark-matter-only simulation to a substantial (kpc-scale) core. The simulated ultrafaint galaxy $\left(M_{\star} \sim 2 \times 10^{4} \mathrm{M}_{\odot}\right)$ does not form enough stars to modify its dark matter halo at all, providing further support to the idea that there is a critical mass below which core formation caused by stellar feedback is energetically impossible (see e.g. Governato et al. 2012; Garrison-Kimmel et al. 2013; Madau, Shen \& Governato 2014). Our results indicate that stellar mass is not the only parameter in core creation, however. The creation of dark matter cores is linked with late-time star formation properties, as only the system with significant late-time star formation forms a sizeable core. The galaxy that forms most of its stars at early time is able to create a core temporarily, but subsequent dark matter accretion and mergers and the lack of strong star formation erase this core, leaving a cuspier profile. The difference in density at $300 \mathrm{pc}$ between these two extreme cases is a factor of $\approx 4$. A related point is that the formation of stable dark matter cores is a continuous process, not instantaneous, and that the creation of significant cores in dwarf galaxies does not appear to be an inevitable outcome in models with bursty star formation histories.

A question that remains unclear is whether these cored systems can avoid regenerating a density cusp once they merge with smaller, cuspier haloes (Laporte \& Peñarrubia 2015). The late-time merger history of dwarfs can vary significantly (e.g. Deason, Wetzel \& 
Garrison-Kimmel 2014), meaning it is imperative to simulate a statistical sample of haloes at a given mass to fully understand trends in core creation or cusp regrowth (Fitts et al., in preparation). It will also be imperative to test this scenario at different halo masses (Chan et al., 2015), as many models predict a core formation efficiency that varies with the halo mass (e.g. Di Cintio et al. 2014). We have not considered the effects stripping from ram pressure and tides that may be important for some Milky Way subhaloes (Read et al. 2006; Zolotov et al. 2012; Brooks \& Zolotov 2014). However, the central prediction coming from our simulations is observationally testable: the presence of cores in galaxies with stellar masses of $\sim 10^{6}-10^{7} \mathrm{M}_{\odot}$ requires substantial late time star formation.

\section{ACKNOWLEDGEMENTS}

This work used computational resources granted by NASA Advanced Supercomputing (NAS) Division, NASA Center for Climate Simulation, Teragrid, and by the Extreme Science and Engineering Discovery Environment (XSEDE), which is supported by National Science Foundation grant number OCI-1053575. JO and JSB were supported by NSF grant AST-1009999 and NASA grant NNX09AG01G. JO thanks the financial support of the Fulbright/MICINN Program. JO also thanks the PYNBODY team for making this software publicly available. DK was supported by a Hellman Fellowship and NSF grant AST-1412153. C-AF-G was supported by NSF through grant AST-1412836, by NASA through grant NNX15AB22G, and by Northwestern University funds.

\section{REFERENCES}

Agertz O. et al., 2007, MNRAS, 380, 963

Amorisco N. C., Zavala J., de Boer T. J. L., 2014, ApJ, 782, L39

Bovill M. S., Ricotti M., 2011, ApJ, 741, 18

Boylan-Kolchin M., Bullock J. S., Kaplinghat M., 2011, MNRAS, 415, L40 Boylan-Kolchin M., Bullock J. S., Kaplinghat M., 2012, MNRAS, 422, 1203

Brook C. B., Di Cintio A., Knebe A., Gottlöber S., Hoffman Y., Yepes G., Garrison-Kimmel S., 2014, ApJ, 784, L14

Brooks A. M., Zolotov A., 2014, ApJ, 786, 87

Brown T. M. et al., 2014, ApJ, 796, 91

Bryan G. L., Norman M. L., 1998, ApJ, 495, 80

Bullock J. S., Kravtsov A. V., Weinberg D. H., 2000, ApJ, 539, 517

Bullock J. S., Dekel A., Kolatt T. S., Kravtsov A. V., Klypin A. A., Porciani C., Primack J. R., 2001, ApJ, 555, 240

Burkert A., 2015, ApJ, 808, 158

Chan T. K., Oñorbe J., Hopkins P. F., Muratov A. L., Faucher-Giguère C.-A., Quataert E., 2015, MNRAS, preprint (arXiv:1507.02282)

Cole A. A., Weisz D. R., Dolphin A. E., Skillman E. D., McConnachie A. W., Brooks A. M., Leaman R., 2014, ApJ, 795, 54

Collins M. L. M. et al., 2014, ApJ, 783, 7

Davis A. J., Khochfar S., Dalla Vecchia C., 2014, MNRAS, 443, 985

Deason A., Wetzel A., Garrison-Kimmel S., 2014, ApJ, 794, 115

de Blok W. J. G., Walter F., Brinks E., Trachternach C., Oh S.-H., Kennicutt R. C., Jr, 2008, AJ, 136, 2648

Dekel A., Silk J., 1986, ApJ, 303, 39

Di Cintio A., Brook C. B., Macciò A. V., Stinson G. S., Knebe A., Dutton A. A., Wadsley J., 2014, MNRAS, 437, 415

Diemand J., Kuhlen M., Madau P., 2007, ApJ, 667, 859

Diemer B., More S., Kravtsov A. V., 2013, ApJ, 766, 25

Donato F. et al., 2009, MNRAS, 397, 1169

Elbert O. D., Bullock J. S., Garrison-Kimmel S., Rocha M., Oñorbe J., Peter A. H. G., 2015, MNRAS, 453, 29

Faucher-Giguère C.-A., Lidz A., Zaldarriaga M., Hernquist L., 2009, ApJ, 703, 1416

Faucher-Giguère C.-A., Hopkins P. F., Kereš D., Muratov A. L., Quataert E., Murray N., 2015, MNRAS, 449, 987
Ferrero I., Abadi M. G., Navarro J. F., Sales L. V., Gurovich S., 2012, MNRAS, 425, 2817

Flores R. A., Primack J. R., 1994, ApJ, 427, L1

Gallazzi A., Charlot S., Brinchmann J., White S. D. M., Tremonti C. A., 2005, MNRAS, 362, 41

Garrison-Kimmel S., Rocha M., Boylan-Kolchin M., Bullock J. S., Lally J., 2013, MNRAS, 433, 3539

Garrison-Kimmel S., Boylan-Kolchin M., Bullock J. S., Lee K., 2014a, MNRAS, 438, 2578

Garrison-Kimmel S., Boylan-Kolchin M., Bullock J. S., Kirby E. N., 2014b, MNRAS, 444, 222

Governato F. et al., 2010, Nature, 463, 203

Governato F. et al., 2012, MNRAS, 422, 1231

Governato F. et al., 2015, MNRAS, 448, 792

Hahn O., Abel T., 2011, MNRAS, 415, 2101

Hoeft M., Yepes G., Gottlöber S., Springel V., 2006, MNRAS, 371, 401

Hopkins P. F., 2013, MNRAS, 428, 2840

Hopkins P. F., 2015, MNRAS, 450, 53

Hopkins P. F., Quataert E., Murray N., 2012, MNRAS, 421, 3522

Hopkins P. F., Narayanan D., Murray N., Quataert E., 2013, MNRAS, 433, 69

Hopkins P. F., Kereš D., Oñorbe J., Faucher-Giguère C.-A., Quataert E., Murray N., Bullock J. S., 2014a, MNRAS, 445, 581

Horiuchi S., Humphrey P. J., Oñorbe J., Abazajian K. N., Kaplinghat M., Garrison-Kimmel S., 2014b, Phys. Rev. D, 89, 025017

Katz N., White S. D. M., 1993, ApJ, 412, 455

Kim J.-h. et al., 2014, ApJS, 210, 14

Kirby E. N., Cohen J. G., Guhathakurta P., Cheng L., Bullock J. S., Gallazzi A., 2013, ApJ, 779, 102

Kirby E. N., Bullock J. S., Boylan-Kolchin M., Kaplinghat M., Cohen J. G., 2014, MNRAS, 439, 1015

Klypin A., Kravtsov A. V., Valenzuela O., Prada F., 1999, ApJ, 522, 82

Klypin A., Karachentsev I., Makarov D., Nasonova O., 2014, MNRAS, preprint (arXiv:1405.4523)

Knollmann S. R., Knebe A., 2009, ApJS, 182, 608

Komatsu E. et al., 2011, ApJS, 192, 18

Kroupa P., 2002, Science, 295, 82

Krumholz M. R., Gnedin N. Y., 2011, ApJ, 729, 36

Kuzio de Naray R., McGaugh S. S., de Blok W. J. G., 2008, ApJ, 676, 920

Laporte C. F. P., Peñarrubia J., 2015, MNRAS, 449, L90

Leitherer C. et al., 1999, ApJS, 123, 3

Macciò A. V., Fontanot F., 2010, MNRAS, 404, L16

Madau P., Shen S., Governato F., 2014, ApJ, 789, L17

Munshi F. et al., 2013, ApJ, 766, 56

Muratov A. L., Keres D., Faucher-Giguere C.-A., Hopkins P. F., Quataert E., Murray N., 2015, MNRAS, preprint (arXiv:1501.03155)

Navarro J. F., Eke V. R., Frenk C. S., 1996, MNRAS, 283, L72

Ogiya G., Burkert A., 2015, MNRAS, 446, 2363

Ogiya G., Mori M., 2014, ApJ, 793, 46

Oh S.-H., de Blok W. J. G., Walter F., Brinks E., Kennicutt R. C., Jr, 2008, AJ, 136, 2761

Oñorbe J., Garrison-Kimmel S., Maller A. H., Bullock J. S., Rocha M., Hahn O., 2014, MNRAS, 437, 1894

Papastergis E., Giovanelli R., Haynes M. P., Shankar F., 2015, A\&A, 574, A113

Peñarrubia J., Pontzen A., Walker M. G., Koposov S. E., 2012, ApJ, 759, L42

Pontzen A., Governato F., 2012, MNRAS, 421, 3464

Porter D. H., 1985, PhD thesis, California University, Berkeley

Power C., Navarro J. F., Jenkins A., Frenk C. S., White S. D. M., Springel V., Stadel J., Quinn T., 2003, MNRAS, 338, 14

Read J. I., Wilkinson M. I., Evans N. W., Gilmore G., Kleyna J. T., 2006, MNRAS, 367, 387

Ricotti M., Gnedin N. Y., 2005, ApJ, 629, 259

Rocha M., Peter A. H. G., Bullock J. S., Kaplinghat M., Garrison-Kimmel S., Oñorbe J., Moustakas L. A., 2013, MNRAS, 430, 81

Sales L. V., Navarro J. F., Schaye J., Dalla Vecchia C., Springel V., Booth C. M., 2010, MNRAS, 409, 1541 
Salucci P., Wilkinson M. I., Walker M. G., Gilmore G. F., Grebel E. K., Koch A., Frigerio Martins C., Wyse R. F. G., 2012, MNRAS, 420, 2034

Sawala T., Guo Q., Scannapieco C., Jenkins A., White S., 2011, MNRAS, 413,659

Sawala T. et al., 2014, MNRAS, preprint (arXiv:1406.6362)

Sawala T. et al., 2015, MNRAS, 448, 2941

Shen S., Madau P., Conroy C., Governato F., Mayer L., 2014, ApJ, 792, 99

Sijacki D., Vogelsberger M., Kereš D., Springel V., Hernquist L., 2012, MNRAS, 424, 2999

Simpson C. M., Bryan G. L., Johnston K. V., Smith B. D., Mac Low M.-M., Sharma S., Tumlinson J., 2013, MNRAS, 432, 1989

Skillman E. D. et al., 2014, ApJ, 786, 44

Springel V., 2005, MNRAS, 364, 1105

Strigari L. E., Frenk C. S., White S. D. M., 2014, preprint (arXiv:1406.6079)

Teyssier R., Pontzen A., Dubois Y., Read J. I., 2013, MNRAS, 429, 3068

Tollerud E. J., Boylan-Kolchin M., Bullock J. S., 2014, MNRAS, 440, 3511

Tolstoy E., Hill V., Tosi M., 2009, ARA\&A, 47, 371

Trujillo-Gomez S., Klypin A., Colín P., Ceverino D., Arraki K. S., Primack J., 2015, MNRAS, 446, 1140

Vogelsberger M., Zavala J., Loeb A., 2012, MNRAS, 423, 3740

Vogelsberger M., Zavala J., Simpson C., Jenkins A., 2014, MNRAS, 444, 3684

Walker M. G., Peñarrubia J., 2011, ApJ, 742, 20

Wechsler R. H., Bullock J. S., Primack J. R., Kravtsov A. V., Dekel A., 2002, ApJ, 568, 52

Weisz D. R., Dolphin A. E., Skillman E. D., Holtzman J., Gilbert K. M., Dalcanton J. J., Williams B. F., 2014, ApJ, 789, 147

Wolf J., Martinez G. D., Bullock J. S., Kaplinghat M., Geha M., Muñoz R. R., Simon J. D., Avedo F. F., 2010, MNRAS, 406, 1220

Zolotov A. et al., 2012, ApJ, 761, 71

\section{APPENDIX A: DARK MATTER PROPERTIES AND EVOLUTION IN THE COLLISIONLESS RUN}

To choose the specific dwarf galaxy haloes to resimulate we rely on collisionless simulations. We have taken into account two things: first we wanted them to be cheap in terms of cpu cost and second we wanted them to be representative of the dwarf galaxy halo population. We point to Oñorbe et al. (2014) for a full description of the method. Here we just want to show how the properties of our selected haloes compare with a realistic sample of dwarf galaxy haloes. To generate this sample we run a $L_{\mathrm{box}}=35 \mathrm{Mpc}$ collisionless simulation $\left(512^{3}\right.$ simulations). Fig. A1 shows the spin $(\lambda)$, concentration $\left(V_{\max } / V_{\mathrm{vir}}\right)$, halo formation time $\left(t_{50}\right)$, and virial mass distributions for all the main haloes in this simulation (so excluding subhaloes) with virial masses between $3 \times 10^{9}$ and $3 \times$ $10^{10} \mathrm{M}_{\odot}$. The mass bin sample includes around $\sim 15000$ haloes. Halo spin parameters were calculated using Bullock et al. (2001) definition. In order to estimate the time of formation for each halo, we followed the approach described in Wechsler et al. (2002). We fit the halo accretion histories obtained from the merger trees to an exponential form that depends on one parameter. The halo formation time $t_{50}$ is calculated at the time when the halo reached half of its total mass. The chosen parameters for our ultrafaint and dwarf initial conditions are plotted as a white triangle and a white square, respectively. The exact values can be found in Table 1 . This figure shows that the resimulated haloes picked from our $L_{\mathrm{box}}=7 \mathrm{Mpc}$ box have very typical values of spin and concentration. The reason why our formation time is a bit lower than the standard value is a combination of three factors. First, we preferred to avoid systems with late major mergers events which also help to reduce the cpu cost of the simulation (Oñorbe et al. 2014). The dwarf halo sample
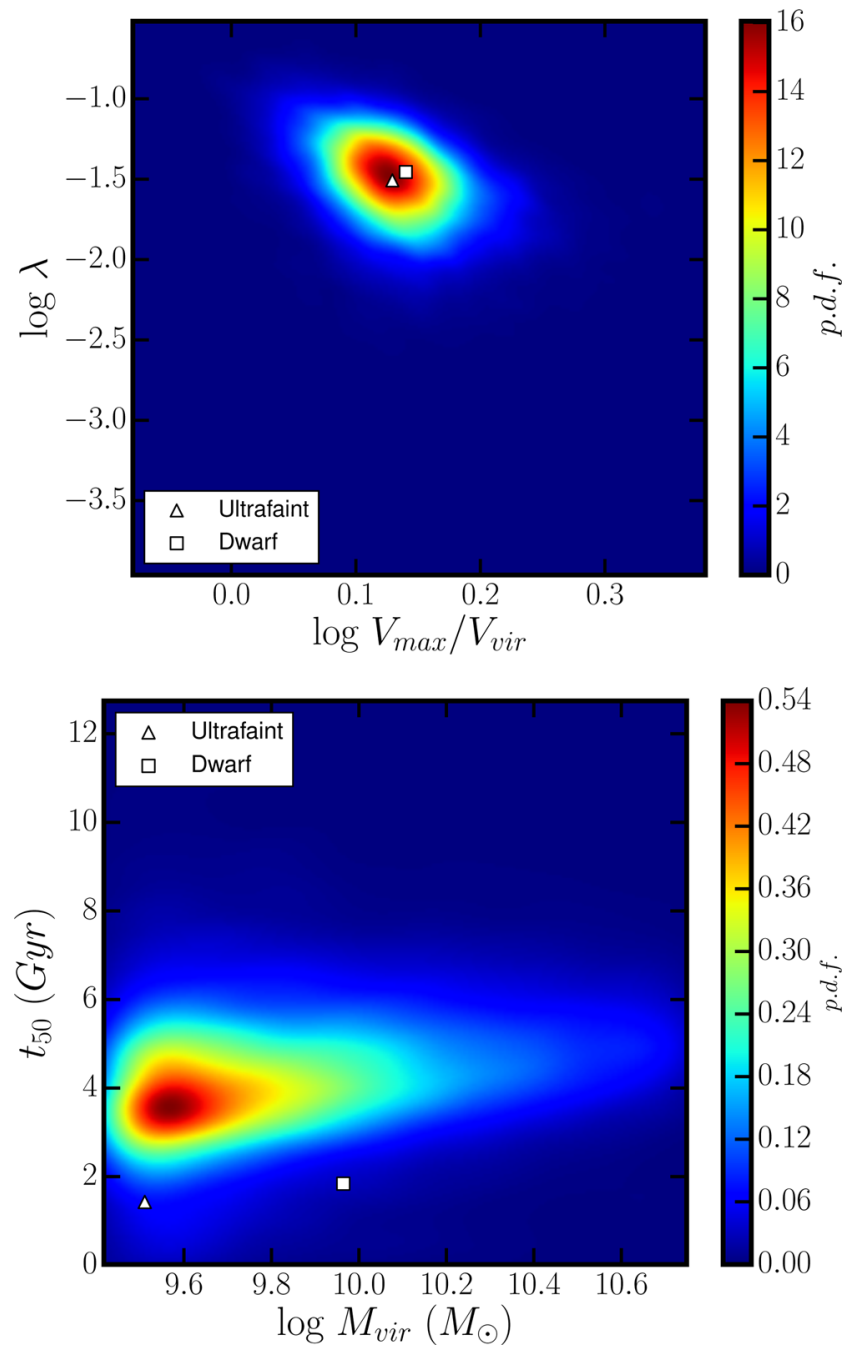

Figure A1. Selecting the sample. Colour map shows the probability density function of the dwarf halo sample in the $L_{\mathrm{box}}=35 \mathrm{Mpc}$ collisionless simulation $\left(512^{3}\right.$ resolution). The white triangle and white square stand for the ULTRAFAINT_DM and DWARF_DM runs, respectively. Upper panel shows concentration versus halo spin. Lower panel shows the virial mass versus halo formation time. Specific values of these parameters can be found in Table 1 .

from a smaller box is biased towards smaller formation times so there were a smaller range of possible haloes to pick which fulfil all our desired criteria. Finally, the circular velocity profile of the DWARF_DM simulation, which can be found in Elbert et al. (2015) (left-hand panel), shows that the halo has too high density to match the circular velocity observations of Local Field dwarf galaxies. This makes it a suitable candidate to study the 'too big to fail' problem.

Fig. A2 shows the evolution of the dark matter mass profile for the DWARF collisionless simulation. Each line shows the amount of dark matter mass contained inside a fixed physical radius. At high redshift the halo shows a characteristic fast halo mass increase followed by a very shallow evolution at high redshift. Notice how the inner parts of the profile take a bit more time to settle down. Below redshift $z \sim 2.5$ the inner part of the halo does not show any significant perturbation as there is no significant accretion or merger (Diemand, Kuhlen \& Madau 2007; Diemer, More \& Kravtsov 2013). 


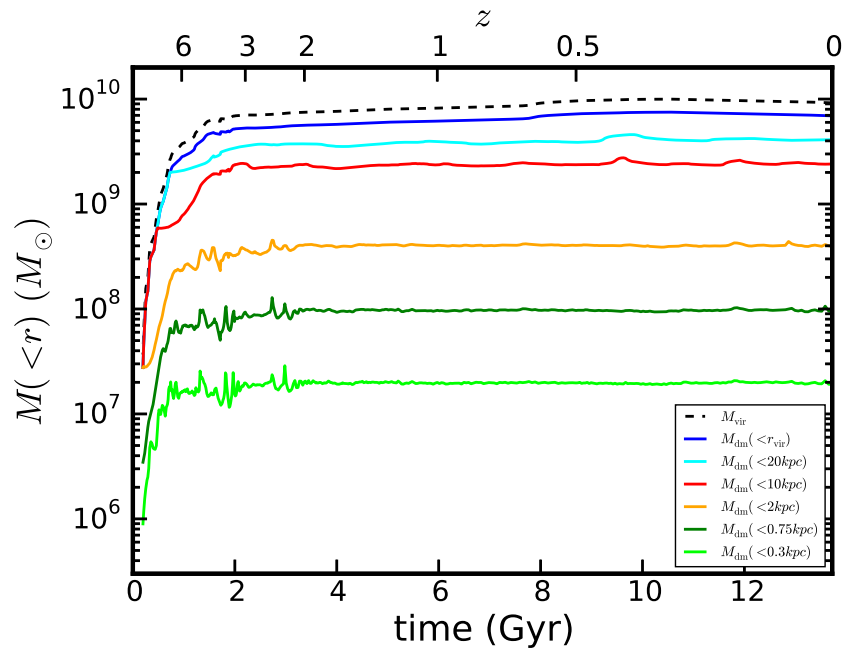

Figure A2. The evolution of the dark matter of the DWARF collisionless run. Each line shows the dark matter mass contained inside a fixed physical radius.

\section{APPENDIX B: CONVERGENCE}

In this section we present a convergence study that we have performed for the DWARF galaxy halo. We have run a lower resolution version of all the DWARF hydrodynamical runs discussed above. The only difference between the high- and low-resolution runs are the different particle masses and softenings used in the runs. The star formation density threshold, $n_{\mathrm{sf}}$, is also slightly different between the runs, $10 \mathrm{~cm}^{-3}$ for low resolution and $100 \mathrm{~cm}^{-3}$ for high resolution. All other code and physical parameters are exactly the same as for the high-resolution runs. In Table B1 all the relevant parameters of these lower resolution runs can be found.

The different panels of Fig. B1 illustrate the differences between the runs. The main difference that we found is that the low-resolution runs have slightly higher stellar masses (upper left-hand panel of Fig. B1). This can be understood by looking at the SFR histories (upper right-hand panel in Fig. B1, blue lines stand for low-resolution runs and red lines for the high-resolution ones). The main difference observed between resolutions is the steeper slope of the cumulative star formation history at lower redshift. This produces higher stellar masses at $z=0$ for the lower resolution runs. We think that this is because the minimum amount of star formation that is possible is set by the gas particle resolution. Therefore the minimum amount of star formation is higher in the lower resolution runs. It is remarkable that all galaxy trends with size and metallicity hold regarding of resolution, so the galaxies seems just move along these relations (lower left-hand panel of Fig. B1). We have also rerun our dwarf galaxy low-resolution initial conditions using exactly the same code to check for pure stochastic differences. The scatter found in all the properties studied in this paper was similar to the one that we found when we change the feedback implementation and/or the softening values. These authors suspect that these differences will decrease

Table B1. Simulations data for the low-resolution convergence tests. First column stands for the different parameters studied for each simulation. In columns 2-9 results for the simulations presented in this work are shown. Row (1): dark matter particle mass in the high-resolution region in $\mathrm{M}_{\odot}$. Row (2): fixed gravitational softening used for the dark matter particles in physical pc. Row (3): baryon particle mass in the high-resolution region in $\mathrm{M}_{\odot}$. Row (4): minimum baryonic force softening in pc (minimum SPH smoothing lengths are comparable or smaller). Recall, force softenings are adaptive (mass resolution is fixed). Row (5): virial mass in $\mathrm{M}_{\odot}$ defined at the overdensity at which the spherical top hat model predicts virialization (Bryan \& Norman 1998). Row (6): maximum circular velocity in $\mathrm{km} \mathrm{s}^{-1}$. Row (7): virial radius in $\mathrm{kpc}$. Row (8): virial baryon fraction, i.e. baryon mass inside the virial radius over the virial mass. Row (9): virial gas fraction, i.e. gas mass inside the virial radius over the virial mass. Row (10): virial stellar fraction, i.e. stellar mass inside the virial radius over the virial mass. Row (11): stellar mass in $\mathrm{M}_{\odot}$. This is the stellar mass of the central galaxy. Row (12): effective stellar mass radius, i.e. half-stellar mass radius in kpc. Row (13): stellar iron over hydrogen ratio. Mass-weighted iron over hydrogen ratio for the dwarf stellar mass component. Row (14): total mass inside $500 \mathrm{pc}$ in $\mathrm{M}_{\odot}$. Row (15): dark matter mass inside $500 \mathrm{pc}$ in $\mathrm{M}_{\odot}$. Row (16): baryon mass inside $500 \mathrm{pc}$ in $\mathrm{M}_{\odot}$. Row (17): gas mass inside $500 \mathrm{pc}$ in $\mathrm{M}_{\odot}$. Row (18): stellar mass inside $500 \mathrm{pc}$ in $\mathrm{M}_{\odot}$.

\begin{tabular}{|c|c|c|c|c|}
\hline Parameter & $\begin{array}{l}\text { DWARF_DM_LR } \\
\text { (Collisionless) }\end{array}$ & $\begin{array}{l}\text { DWARF_LATE_LR } \\
\text { (Hydro: Feed-M) }\end{array}$ & $\begin{array}{l}\text { DWARF_MIDDLE_LR } \\
\text { (Hydro: Feed-M-soft) }\end{array}$ & $\begin{array}{l}\text { DWARF_EARLY_LR } \\
\text { (Hydro: Feed-V) }\end{array}$ \\
\hline (1) $m_{\mathrm{p}}^{\mathrm{dm}}\left(\mathrm{M}_{\odot}\right)$ & $1.21 \times 10^{4}$ & $1.01 \times 10^{4}$ & $1.01 \times 10^{4}$ & $1.01 \times 10^{4}$ \\
\hline (2) $\epsilon_{\mathrm{dm}}(\mathrm{pc})$ & 35 & 35 & 35 & 35 \\
\hline (3) $m_{\mathrm{p}}^{\mathrm{bar}}\left(\mathrm{M}_{\odot}\right)$ & - & $2.04 \times 10^{3}$ & $2.04 \times 10^{3}$ & $2.04 \times 10^{3}$ \\
\hline (4) $\epsilon_{\mathrm{gas}}^{\mathrm{pm}}(\mathrm{pc})$ & - & 2.0 & 35 & 2.0 \\
\hline (5) $M_{\text {vir }}\left(\mathrm{M}_{\odot}\right)$ & $9.48 \times 10^{9}$ & $7.60 \times 10^{9}$ & $7.60 \times 10^{9}$ & $7.46 \times 10^{9}$ \\
\hline (6) $V_{\max }\left(\mathrm{km} \mathrm{s}^{-1}\right)$ & 37.31 & 32.79 & 32.79 & 33.56 \\
\hline (7) $r_{\mathrm{vir}}(\mathrm{kpc})$ & 54.99 & 51.08 & 51.08 & 50.77 \\
\hline (8) $f_{\text {bar }}$ & - & 0.0166 & 0.0171 & 0.0137 \\
\hline (9) $f_{\text {gas }}$ & - & 0.0160 & 0.0165 & 0.0121 \\
\hline (10) $f_{*}$ & - & 0.0006 & 0.0006 & 0.0016 \\
\hline$(11) M_{*}\left(\mathrm{M}_{\odot}\right)$ & - & $4.1 \times 10^{6}$ & $4.2 \times 10^{6}$ & $1.0 \times 10^{7}$ \\
\hline$(12) r_{1 / 2}^{*}(\mathrm{kpc})$ & - & 0.783 & 0.881 & 1.311 \\
\hline (13) $[\mathrm{Fe} / \mathrm{H}]$ & - & -1.493 & -1.468 & -1.450 \\
\hline$(14) M_{500}^{\mathrm{tot}}\left(\mathrm{M}_{\odot}\right)$ & $3.212 \times 10^{7}$ & $1.285 \times 10^{7}$ & $1.014 \times 10^{7}$ & $4.088 \times 10^{6}$ \\
\hline$(15) M_{500}^{\mathrm{dark}}\left(\mathrm{M}_{\odot}\right)$ & $2.414 \times 10^{7}$ & $7.366 \times 10^{6}$ & $7.132 \times 10^{6}$ & $3.703 \times 10^{6}$ \\
\hline (16) $M_{500}^{\mathrm{bar}}\left(\mathrm{M}_{\odot}\right)$ & - & $5.483 \times 10^{6}$ & $3.002 \times 10^{6}$ & $3.861 \times 10^{5}$ \\
\hline (17) $M_{500}^{\text {gas }}\left(\mathrm{M}_{\odot}\right)$ & - & $5.083 \times 10^{6}$ & $2.635 \times 10^{6}$ & 0.0 \\
\hline$(18) M_{500}^{*}\left(\mathrm{M}_{\odot}\right)$ & - & $4.004 \times 10^{5}$ & $3.677 \times 10^{5}$ & $3.861 \times 10^{5}$ \\
\hline
\end{tabular}



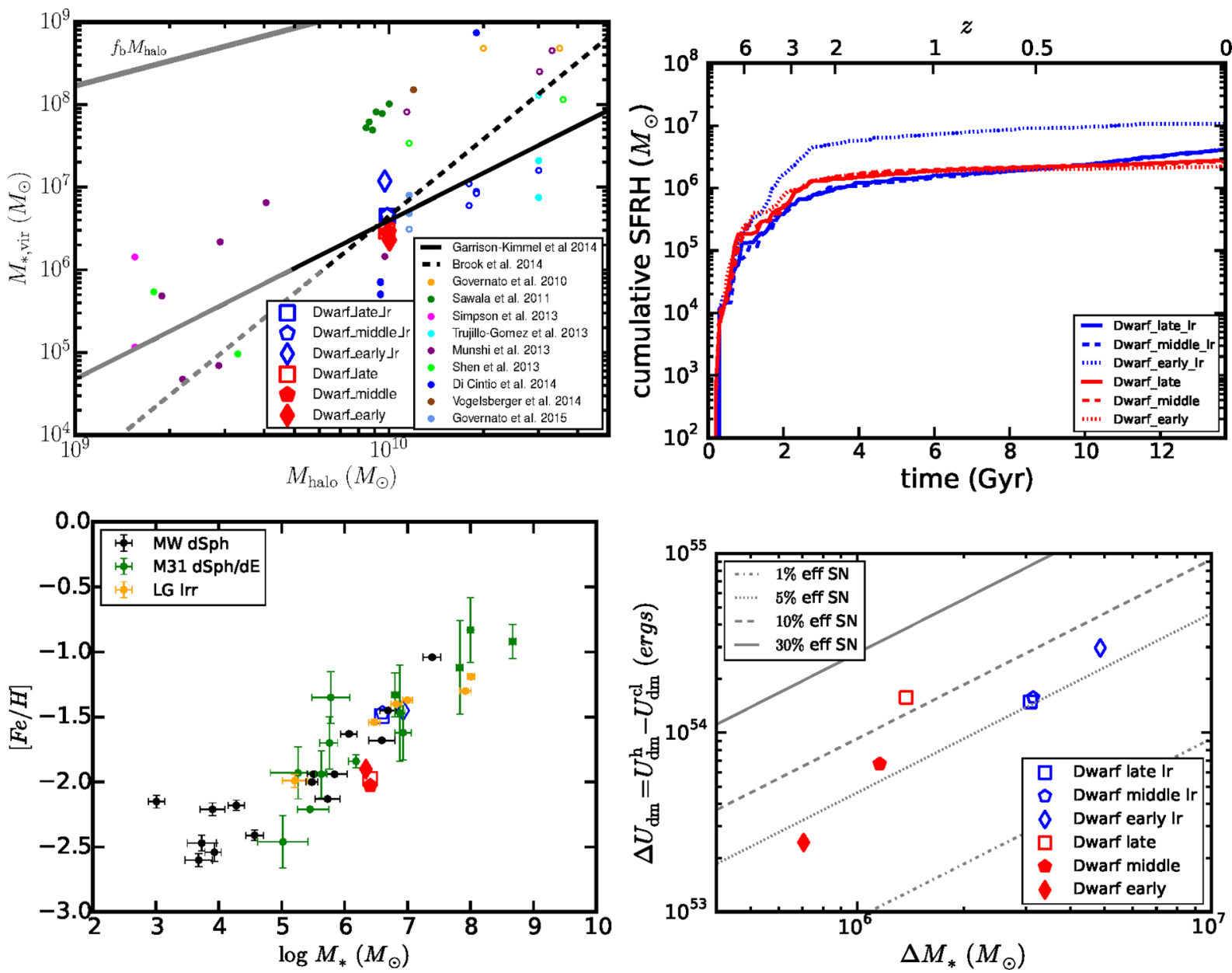

Figure B1. Convergence tests. High (red) and low (blue) resolution simulations. Upper left: the stellar mass-halo mass relation. Upper right: cumulative star formation history. Lower left: metallicity versus stellar mass. Lower right: energy considerations in the formation of the dark matter cores in a $10^{10} \mathrm{M}_{\odot}$ halo. See text for details.

at higher resolution, though higher resolution runs will certainly be required in order to test this conjecture.

Finally, concerning the core formation and energy considerations, low-resolution runs also form a core which seems to be directly connected with its SFR at low redshifts $(z \lesssim 2)$. In general these cores are more prominent than their high-resolution counterparts due to the higher SFR discussed above. Remarkably when we plot the energy requirements to form these cores versus the amount of energy obtained from SN feedback below $z=2$ (lower right-hand panel of Fig. B1), they lie in the same range of efficiencies as their high-resolution counterparts.
Although it is not possible to claim full convergence for our high-resolution runs from these results, we think that they are at least quite encouraging and definitely an improvement from other approaches in which parameters of the subgrid physics must be tuned at each resolution.

This paper has been typeset from a $\mathrm{T}_{\mathrm{E}} \mathrm{X} / \mathrm{L} \mathrm{T}_{\mathrm{E}} \mathrm{X}$ file prepared by the author. 